\title{
Effect of Thermal Boundary Conditions on Propagation of Non- Equidiffusive Flames in Pipes
}

Swathi Reddy Shetty

Follow this and additional works at: https://researchrepository.wvu.edu/etd

\section{Recommended Citation}

Reddy Shetty, Swathi, "Effect of Thermal Boundary Conditions on Propagation of Non-Equidiffusive Flames in Pipes" (2017). Graduate Theses, Dissertations, and Problem Reports. 6488.

https://researchrepository.wvu.edu/etd/6488

This Thesis is protected by copyright and/or related rights. It has been brought to you by the The Research Repository @ WVU with permission from the rights-holder(s). You are free to use this Thesis in any way that is permitted by the copyright and related rights legislation that applies to your use. For other uses you must obtain permission from the rights-holder(s) directly, unless additional rights are indicated by a Creative Commons license in the record and/ or on the work itself. This Thesis has been accepted for inclusion in WVU Graduate Theses, Dissertations, and Problem Reports collection by an authorized administrator of The Research Repository @ WVU. For more information, please contact researchrepository@mail.wvu.edu. 


\title{
Effect of Thermal Boundary Conditions on Propagation of Non-Equidiffusive Flames in Pipes
}

\author{
Swathi Reddy Shetty
}

\author{
Thesis submitted to the \\ Benjamin M. Statler College of Engineering and Mineral Resources \\ at West Virginia University \\ in partial fulfillment of the requirements for the degree of \\ Master of Science \\ in Mechanical Engineering \\ Dr. V'yacheslav Akkerman, Ph.D., Chair \\ Dr. Patrick Browning, Ph.D., \\ Dr. Yogendra Panta, Ph.D.
}

Department of Mechanical and Aerospace Engineering

\author{
Morgantown, West Virginia \\ 2016
}

Keywords: combustion, 2D channels, adiabatic, isothermal, thermal expansion, flame acceleration, Flame propogation, Flame front, non-equdiffusive flames, Lewis number,

Reynolds number, Markstein number, Computational and analytical formulations

Copyright 2016 Swathi Reddy Shetty 


\section{Abstract \\ Effect of thermal boundary conditions on propagation of non-equidiffusive flames in pipes Swathi Reddy Shetty}

Boundary conditions constitute one of the key factors influencing combustion in chambers with large aspect ratios such as narrow channels or pipes. Specifically, the flame shape and propagation velocity are impacted by wall friction and heat transfer to the walls. Both factors continuously deform the flame front, thereby resulting in its larger surface area as compared to a planar flame front. Such a corrugated flame consumes more fuel per unit time and thereby propagates faster than the planar flame at the same thermal-chemical conditions. Consequently, a flame accelerates due to the boundary conditions.

In the recent years, there have been many studies scrutinizing the role of boundary conditions in the flame acceleration scenario by means of analytical formulations, computational endeavors and experimental measurements. However, the majority of these works was limited to equidiffusive flames, where the thermal-to-mass diffusivity ratio (the Lewis number; Le) is unity. In this respect, the present thesis removes this limitation by analyzing non-equidiffusive (fuel-lean, Le $<1$, and rich, Le > 1) flames propagating in pipes of various widths. Specifically, a parametric study has been conducted by means of simulations of the basic hydrodynamic and combustion equations.

A fully-compressible Navier-Stokes solver available in Akkerman's group at West Virginia University was employed in the simulations. The embryo of this solver was originally developed at Volvo Aero / Chalmers University, Sweden, and then upgraded by various research groups and adapted for parallel computations. In this study, specifically, two-dimensional (2D) channels with smooth walls and different thermal conditions such as isothermal and adiabatic walls, have been employed for various Lewis numbers in the range $0.2 \leq \mathrm{Le} \leq 2.0$, and the Reynolds number associated with the flame propagation in the range $5 \leq \operatorname{Re} \leq 30$.

As a result, a strong coupling between the wall conditions and the variations of the Lewis and Reynolds numbers is demonstrated. Specifically, it is observed that the increase in the Lewis number results in the moderation of flame tip acceleration. It is also found that there is a change in the burning rate and surface area of the flame front at the lower Lewis numbers, where flames appear unstable against the thermal-diffusion instability. Moreover, a very substantial difference between the situations of isothermal and adiabatic wall conditions is demonstrated. 


\section{Acknowledgements}

I would like to thank all the individuals without whom this project would not have been possible and also everyone who have been a great source of support and encouragement during the impossible times.

Specifically, the first person I would like to thank is Dr. V'yacheslav Akkerman. He has been a great mentor and professor to me since I came to West Virginia University. He is the sole reason for my successful completion this phase of my education, and without his patience, direction and support, I would not have completed this project.

I would also like to extend my sincere thanks to my committee members for making time for me on a short notice and helping me. A special thanks to Dr. Patrick Browing for being my former advisor and guiding me.

My gratitude to my research group peers: Sinan Demir, Afeez Adebiyi and Bugzy Idowu. Specifically, thank you, Sinan, for being my guide and teaching me everything I need to know as well as encouraging me not to give up. Without your support and help I would not have completed my degree.

Finally, I would like to extend my sincere acknowledgement to my family and all my friends who have always been there for me. Their love, support and encouragement have made me the individual I am. 


\section{Dedication}

I dedicate this work as any other product of mine to my parents and sister, without whose love, support and encouragement I could not have accomplished so far 


\section{Table of Contents}

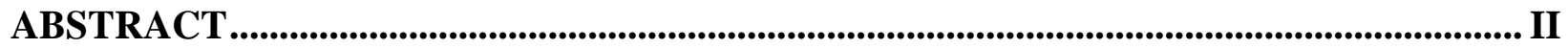

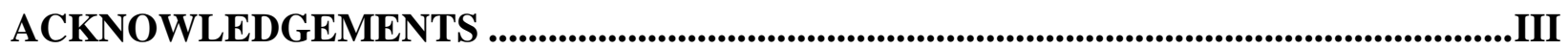

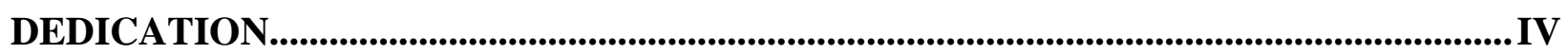

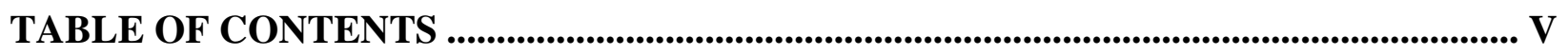

NOMENCLATURE..................................................................................................... VII

LIST OF FIGURES ................................................................................................................................... IX

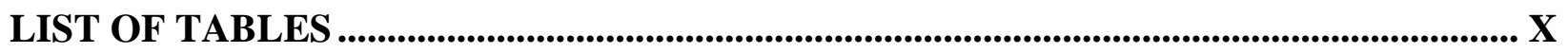

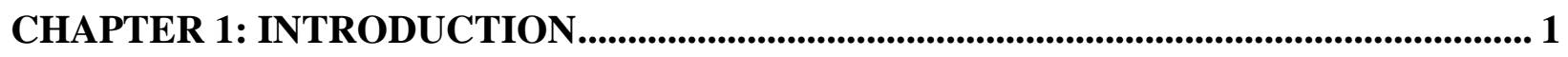

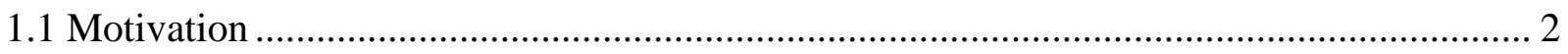

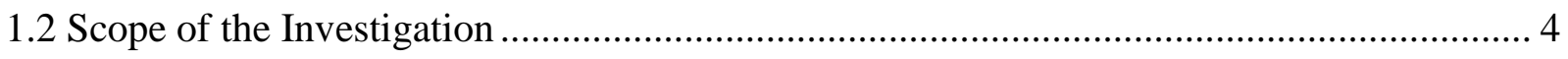

CHAPTER 2: LITERATURE SURVEY ....................................................................................... 5

CHAPTER 3: METHODOLOGY .............................................................................................. 9

3.1 Numerical Approach ............................................................................................ 10

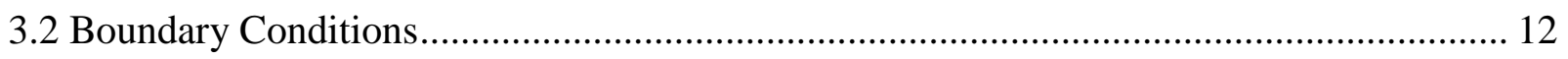

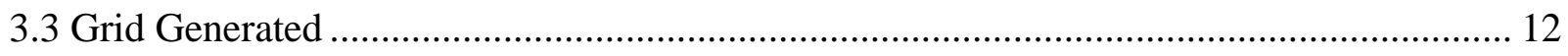

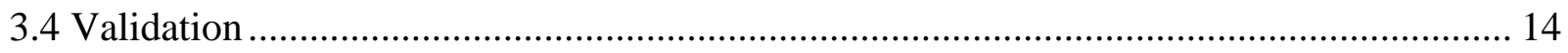

CHAPTER 4: THEORY ........................................................................................................................... 155

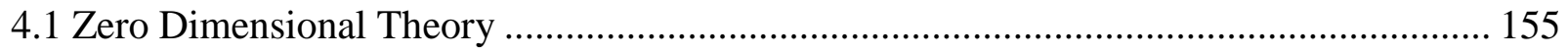

4.2 Theory of Flame Acceleration in 2D Channels ............................................................... 166

4.3 Flame Acceleration in Adiabatic and Isothermal Wall Conditions.................................... 177

CHAPTER 5: RESULTS AND DISCUSSION ................................................................ 19 
5.1 Equidiffusive Flames with Adiabatic Walls.......................................................... 200

5.2 Le <1 Flames with Adiabatic Walls ...................................................................... 244

5.3 Le >1 Flames with Adiabatic Walls .............................................................................. 277

5.4 Isothermal Walls at Low Temperature ................................................................ 29

5.5 Isothermal Walls at High Temperature ............................................................ 322

CHAPTER 6: CONCLUSION............................................................................................... 36

REFERENCES ..................................................................................................................... 38 


\section{Nomenclature}

CV Heat capacity at constant volume

$C P \quad$ Heat capacity at constant pressure

c Speed of the sound

EA Activation energy

H Enthalpy

Lf $\quad$ Flame thickness

$L f^{*} \quad$ Flame thickness, including wall effects

Lc $\quad$ Cell length

$L_{m} \quad$ Markstein Length

m Constant molar mass

Ma Mach number

Mk Markstein number

$P \quad$ Pressure

$\operatorname{Pr} \quad$ Prandtl number

$Q \quad$ Specific energy released in a chemical reaction

$q_{i j} \quad$ Energy diffusion vector

$r \quad$ Radial position

$R \quad$ Flame tip position along the conduit

Rch Half width of the channel

$R c \quad$ Flame tip position at the conduit centerline

Re Reynolds number

$R \mathrm{u} \quad$ Universal gas constant

Rtu Channel width

Sc Schmidt number

$T \quad$ Temperature

Tw Wall temperature

$t \quad$ Time

$u \quad$ Velocity component

$U_{c} \quad$ Flame velocity at the center of the pipe

Uf Planar flame velocity 
$U$ tip Flame tip velocity

$U_{w} \quad$ Flame velocity near the wall

$Y \quad$ Mass fraction of the fuel

$Z \quad$ Axial flame position

Ze Zel'dovich number

\section{Greek Letters}

$\alpha \quad$ Blockage ratio

$\gamma \quad$ Adiabatic exponent

$\gamma_{i j} \quad$ Stress tensor

$\varepsilon \quad$ Total energy per unit volume

$\zeta \quad$ Dynamic viscosity

$\Theta \quad$ Thermal expansion coefficient

$\lambda \quad$ Cell width size

$\rho \quad$ Density

$\sigma \quad$ Acceleration rate

$\tau \quad$ Scaled time

$\tau f \quad$ Scaled time with respect to the flame

$v \quad$ Kinematic viscosity

$\vartheta \quad$ Scaled temperature 


\section{List of Figures}

Figure 1.1: A sketch of premixed flame propagation scenario............................................ 2

Figure 1.2: Applications of channel combustion: a) Nano tube, b) UAV, c) coal mines and d) rotary engine

Figure 2.1: The stages of DDT as described by Zeldovich et al. [12] ..................................... 5

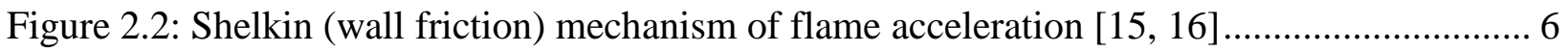

Figure 2.3: Evolution of isotherms for $\mathrm{Re}=25, \mathrm{Pr}=1$ from $600 \mathrm{~K}$ to $2100 \mathrm{~K}$ in steps of $300 \mathrm{~K}$ [15].

Figure 2.4: Experimental setup to capture the propagating flame front [40]............................. 9

Figure 2.5: a) Images of premixed hydrogen-air flame, b) the tulip flame formation and distortion [40]

Figure 3.1: The dynamic mesh with variable resolution [46]. 13

Figure 3.2: A resolution test for the flame tip position $Z_{t i p} / R$ versus the scaled time $S_{L} t / R$ for the different mesh sizes of $L_{f}, 0.5 L_{f}, 0.25 L_{f}$ and $0.125 L_{f}$ with Le $=1$ in each case [21]......... 13

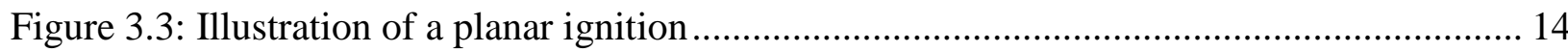

Figure 3.4: Scaled tip velocity vs the scaled time charts with both numerical and experimental results: (a) $\mathrm{R}=0.25 \mathrm{~mm}$; (b) $\mathrm{R}=0.50 \mathrm{~mm}[43]$..... 14

Figure 4.1: $\mathrm{Ze}_{\mathrm{C}}$ as the function of $\Theta$ for $h(\vartheta)=1$ and $h \vartheta=\vartheta 1 / 2$. 166

Figure 4.2: $\mathrm{Le}_{\mathrm{c}}$ as the function of Ze for $h(\vartheta)=1$ shown by solid lines and $h \vartheta=\vartheta 12$ shown by dotted lines. 166

Figure 4.3: $\Theta$ vs Re diagram representing the exponential and non-exponential acceleration regimes. The solid line is the critical line where this transition takes place. [45] 177

Figure 4.4: Flame structure with adiabatic walls 188

Figure 4.5: The isothermal wall conditions. ................................................................. 18

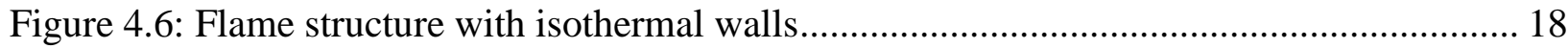

Figure 5.1: Computational thermal model created by the solver. 19

Figure 5.2: The scaled flame tip velocity, $\mathrm{U}_{\text {tip }} / \mathrm{U}_{\mathrm{f}}$, the surface area, $\mathrm{A}_{\mathrm{w}} / \mathrm{D}$, and the scaled total burning rate, $\mathrm{U}_{\mathrm{w}} / \mathrm{U}_{\mathrm{f}}$, versus the scaled time $\tau=\mathrm{U}_{\mathrm{f}} \mathrm{t} / \mathrm{R}$ for $\mathrm{Le}=1$ and $\mathrm{Re}=5$ (a) and 10 (b)... 211 Figure 5.3: The scaled burning rate $U_{w} / U_{f}$ versus the scaled time $\tau=U_{f} t / R$ for different flame

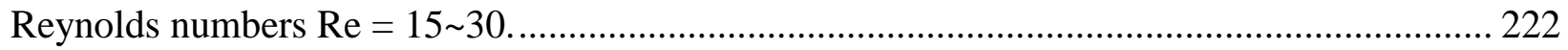


Figure 5.4: The scaled flame surface area $A_{w} / D$ versus the scaled time $\tau=U_{f} t / R$ for different $R e$

Figure 5.5: The scaled flame tip velocity $\mathrm{U}_{\text {tip }} / \mathrm{U}_{\mathrm{f}}$ vs the scaled time $\tau=\mathrm{U}_{\mathrm{f}} \mathrm{t} / \mathrm{R}$ for different $\operatorname{Re} 233$ Figure 5.6: The color temperature snapshots of the flame evolution for $\mathrm{Le}=1$ and $\operatorname{Re}=15 \ldots .233$

Figure 5.7: The color temperature snapshots for the flame evolution for $\mathrm{Le}=1$ and $\operatorname{Re}=25 \ldots 233$

Figure 5.8: The properties of flame front versus scaled time for $\mathrm{Le}=0.2$ and $\mathrm{Re}=20 \ldots \ldots \ldots .244$

Figure 5.9: The color snapshots of the flame evolution for $\mathrm{Le}=0.2$ and $\mathrm{Re}=20 \ldots \ldots \ldots \ldots \ldots . .255$

Figure 5.10: The properties of flame front versus the scaled time for $\mathrm{Le}=0.6$ and $\mathrm{Re}=20 \ldots \ldots . .255$

Figure 5.11: The color snapshots of the flame evolution for $\mathrm{Le}=0.6$ and $\mathrm{Re}=20 \ldots \ldots \ldots \ldots \ldots .266$

Figure 5.12: The color snapshots of the flame evolution for $\mathrm{Le}=0.2$ and $\operatorname{Re}=20 \ldots \ldots \ldots \ldots \ldots . .266$

Figure 5.13: The color snapshots of the flame evolution for $\mathrm{Le}=0.2$ and $\operatorname{Re}=30 \ldots \ldots \ldots \ldots \ldots . .266$

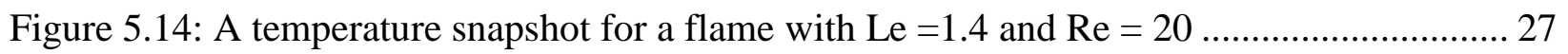

Figure 5.15: A temperature snapshot for a flame with $\mathrm{Le}=2.0$ and $\mathrm{Re}=20 \ldots \ldots \ldots \ldots \ldots \ldots \ldots . . .27$

Figure 5.16: A temperature snapshot for a flame with $\mathrm{Le}=1.4$ and $\mathrm{Re}=30 \ldots \ldots \ldots \ldots \ldots \ldots \ldots \ldots . . . .27$

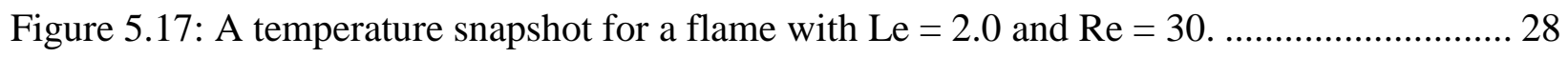

Figure 5.18: The scaled burning rate versus the scaled time for fuel-rich flames ..................... 28

Figure 5.19: The scaled flame properties versus the scaled time for $\mathrm{Le}=1.4$ and $\operatorname{Re}=20 \ldots \ldots . .29$

Figure 5.20: The color snapshots of the flame evolution for Le $=0.2$ and $T_{w}=300 \mathrm{~K} . \ldots \ldots \ldots . . .300$

Figure 5.21: The color snapshots of the flame evolution for Le $=1.4$ and $T_{w}=300 \mathrm{~K} \ldots \ldots \ldots \ldots . . .311$

Figure 5.22: Comparison of the burning rate time evolutions for $T_{w}=300 \mathrm{~K}$ and various Le. . 311

Figure 5.23: The scaled flame tip velocity versus the scaled time for $T_{w}=300 \mathrm{~K}$ and various Le

Figure 5.24: The color snapshots of the flame evolution for Le $=0.2$ at $T_{w}=800 \mathrm{~K}$

Figure 5.25: The scaled burning rate $U_{w} / U_{f}(\mathrm{a})$, the scaled flame surface area $A_{w} / D(\mathrm{~b})$ and the scaled tip velocity $U_{\text {tip }} / U_{f}$ versus the scaled time $\tau=U_{f} t / R$ for various Le $=0.2 \sim 2.0$......... 344

Figure 5.26: Time evolution of the flame properties for Le $=0.6$ with $T_{w}=800 \mathrm{~K}$. 355

\section{List of Tables}

Table 3.1: Basic equations 


\section{Chapter 1: Introduction}

Combustion is a chemical reaction between a fuel and an oxidant (oxidizer) which is highly exothermic in nature. Oftentimes, heat released by such a reaction makes it self-sustainable. Humans have been finding practical uses of combustion such as cooking or heating since the primitive times. With the start of civilization and the discovery of a steam engine, a new usage of combustion - production of mechanical work - has been discovered. The latter is a backbone of the modern civilization.

In fact, steam engines have been used in transportation of railways and ships. With the dawn of fuels like gasoline and its by-products, the internal combustion (IC) engines, which use power of fuels to drive pistons and create movement, appear.

Burning of fuels can be classified as stationary or explosive [1]. Stationary combustion can be observed, in particular, in gasoline lamps with wicks. In contrast, explosive combustion is where a flame moves rapidly through the combustible mixture as occurs in gasoline engines [1].

The burning mixture can be classified as premixed or non-premixed. Specifically, premixed combustion is that where the mixing of the fuel and oxidizer occurs before the ignition. Typical premixed flame propagation is illustrated in Fig 1.1. This can be seen in spark-ignition (SI; Otto) gasoline engines. In non-premixed flames, the fuel and oxidizer are sent into the burning zone

separately where mixing occurs by molecular or turbulent diffusion. An example of this is a compression ignition (CI; Diesel) engine.

The present research is focused on premixed combustion. We can compare the achieved flame velocities with the speed of sound and distinguish the two regimes of premixed burning:

1) Deflagration (or flame): In this case, the reaction is progressed by thermal conduction such that the flame propagation velocity is of the order of 0.1-10 m/s, being thereby strongly subsonic $[2,3]$.

2) Detonation: In this case, the reaction is driven by the shock waves that compress and preheat the fuel mixture, which finally explodes and detonates. Here the flame propagation velocity is supersonic, typically around $2000 \mathrm{~m} / \mathrm{s}[2,3]$. 


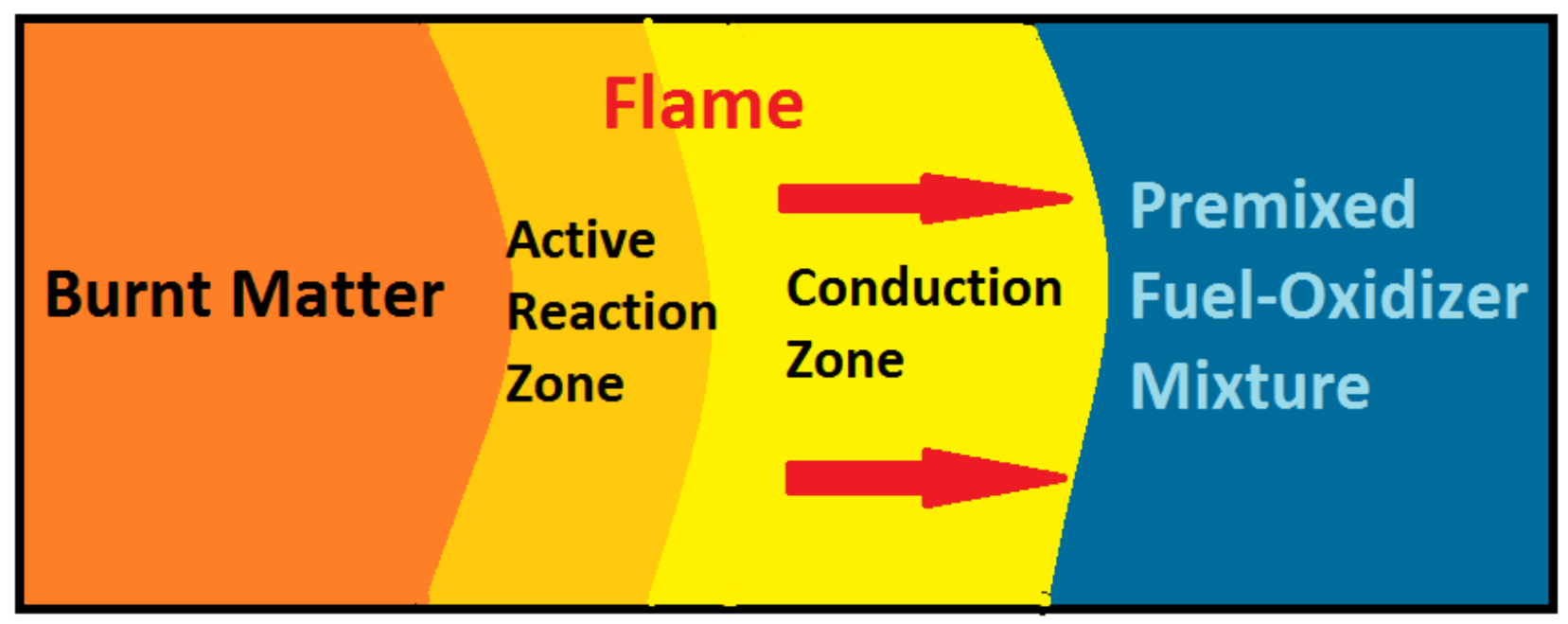

Figure 1.1: A sketch of premixed flame propagation scenario.

By observing characteristic velocities in these regimes one can conclude that detonations propagate 2-4 orders faster than deflagrations. In some cases, a deflagration-to-detonation transition (DDT) event occurs, this is commonly observed in nature also, especially during the explosion of the supernovae stars. In terrestrial conditions, this principle is also the base for the pulse-detonation engine. Concurrently, DDT is dangerous and unpredictable. This is one of the most intriguing problems presently faced by the researchers [3]. Hence the study of DDT has gained importance. Understanding this transition will give us better control over DDT and will be advantageous to minimize the adverse effects in cases where it is not desired.

\subsection{Motivation}

As the need to study combustion is gaining importance, a lot of extensive study has been done on different flame configurations [4] as shown in Fig. 1.2. To simplify the solution the most reasonable and preferably used geometries are pipes (channels or tubes) and slits. This is used not only because of its simplicity, but also because it has many practical applications such as:

1. Pseudo-combustion of thermo-power waves in nanotubes [5] (Fig. 1.2a): Here the nanotube or nanowire of high axial thermal conductivity is coupled with an exothermic chemical reaction to produce a self-propagating reactive wave that can be driven along its length. These waves with high power density may be used for unique energy sources.

2. Micro and mesa combustors [6] (Fig. 1.2b): Micro combustion is gaining importance as 
the world is moving towards smaller length scales. For example, miniature satellites and unmanned aerial vehicles (UAV) have various applications. As the power source should be able to match the geometry, the study of combustion at small scales is important. Mesa-scales are distinguished from "micro" when the heat loss is comparable to the heat generated, thus, mesa-combustors are also gaining importance.

3. Fire safety in mines [7] (Fig. 1.2c): A great hazard is represented by the accidental dust deflagrations to people and equipment in transportation of flammable products. Study of combustion in tubes is essential in case of coalmines, where sudden explosions may result in a lot of risks due to the presence of flammable dust and gases which provoke the combustion.

4. Micro Electric Mechanical Systems (MEMS) [8] (Fig. 1.2d): A lot of research is going on the idea of micro-electro-mechanical-system internal combustion engines. Micro internal combustion engines have been developed to provide compact electric power source and micro vehicle propulsion units.
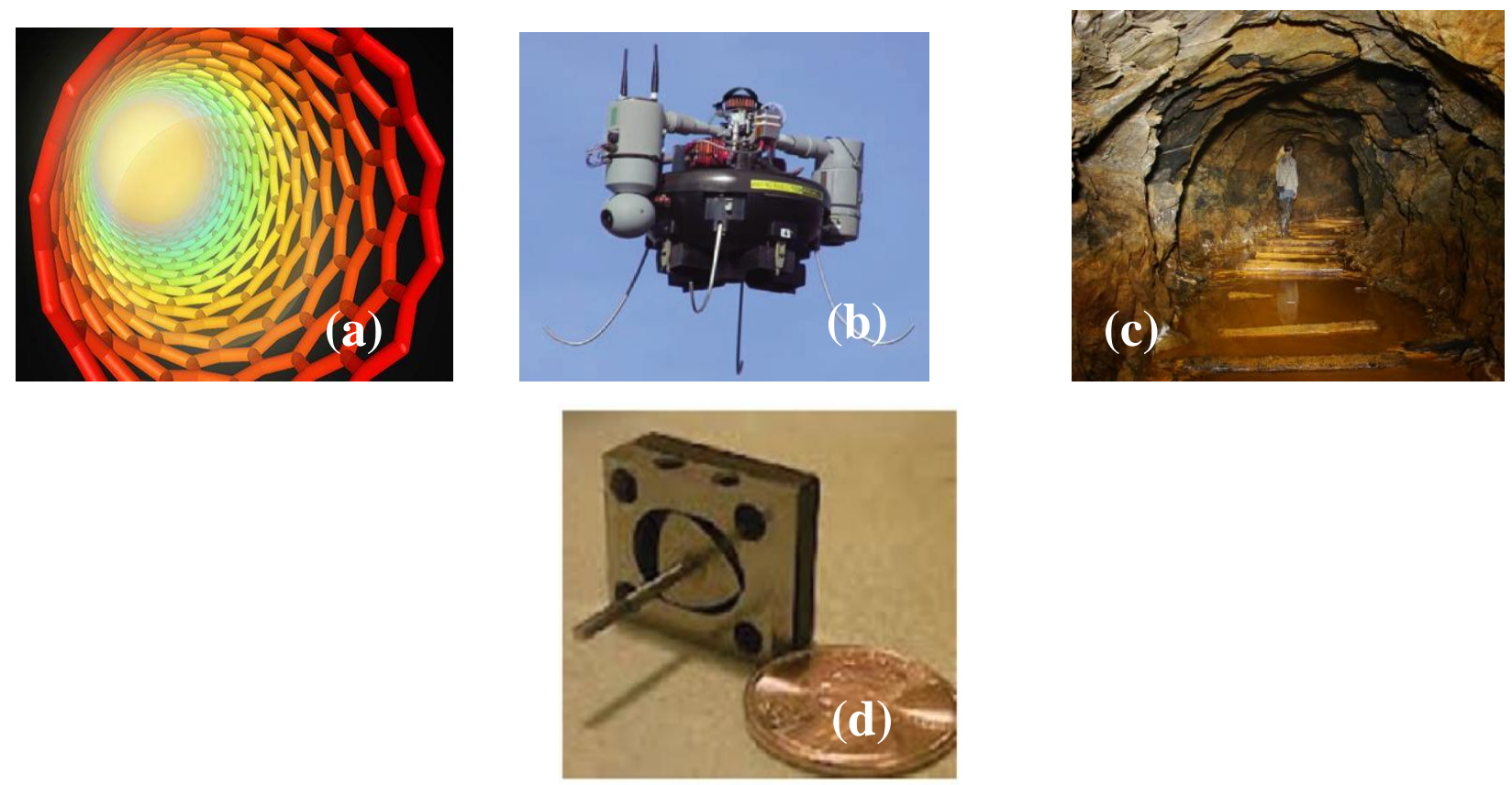

Figure 1.2: Applications of channel combustion: a) Nano tube, b) UAV, c) coal mines and d) rotary engine. 
Therefore, there is an increasing demand to understand dynamics and morphology of flames that flashes researchers' minds in many applications as mentioned above.

\subsection{The Scope of the Investigation}

In this thesis, the effects of thermal boundary conditions on the flame propagation dynamics are scrutinized. With this purpose, a parametric study with the help of computational means has been implemented. Two types of thermal conditions have been used:

1. Adiabatic Wall Conditions: The pipes are subjected to adiabatic conditions, where the system is insulated. Here, the flame morphology and propagation are observed in relation to the changing Le number and the width of the channel (the Reynolds number).

2. Isothermal Wall Conditions: The pipes are subjected to isothermal conditions, where the temperatures of the wall are maintained constant. Here, the flame morphology and propagation are observed in relation to the changing Le number at both low and high temperature walls.

The burning rates, along with the change in surface area of the flame front, characterized by the fuel thermal expansion

$$
\Theta=T_{b} / T_{f}=\rho_{f} / \rho_{b}
$$

have been investigated in both cases, while the scaled tip velocity changes are recorded during the flame propagation. In both cases, we are making the flames non-equidiffusive by changing the Lewis number (Le) defined as the mass diffusivity to the thermal diffusivity ratio. The Lewis number can also be calculated from the ratio of the Schmidt (Sc) and Prandtl (Pr) numbers as:

$$
\mathrm{Le}=\mathrm{Sc} / \mathrm{Pr} \text {. }
$$




\section{Chapter 2: Literature Survey}

Even though combustion applications are employed for centuries unless millennia, there was only little understanding on the subject of flame morphology at a time a fire was first employed. As the centuries passed, the knowledgebase over this topic has been increased tremendously. Various aspects of combustion attracted a lot of researchers. As such the conceptual process leading flame acceleration to detonation has also been studied and showcased in the recent studies $[9,10]$. Accordingly, DDT can occur because of an intense expansion of burning mixture, which pushes and provokes a spontaneous reaction in the fresh fuel mixture ahead of it, because of compression [11]. Zeldovich el al. [12] proposed a DDT scenario as that in Fig. 2.1.

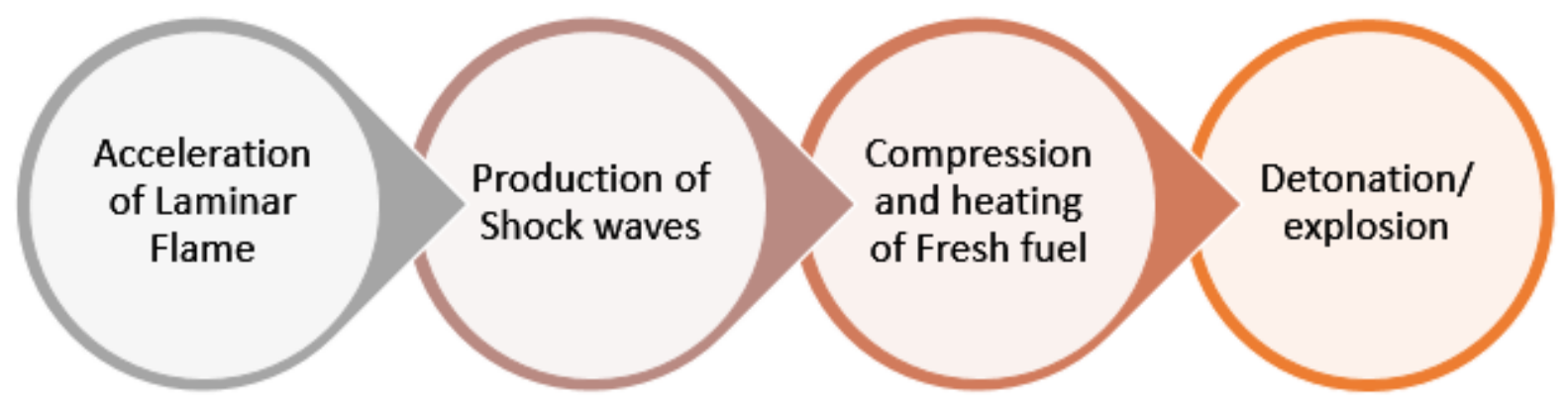

Figure 2.1: The stages of DDT as described by Zeldovich et al. [12].

In general, flame corrugation and following acceleration occur due to many reasons such as combustion instabilities, acoustic effects, turbulence and geometric features [13]. One of the acceleration mechanisms related to the initial stage of the process was first studied by Clanet and Searby [14]. In this mechanism, the flame front develops from a spherical kernel to a fingershaped front during the laminar stage and then accelerates instantaneously. Later analytical formulation on this flame model has been developed by Bychkov et al [15].

Another acceleration mechanism, suggested by Shelkin et al. [16], explains spontaneous acceleration as a result of the non-slip conditions at the pipe walls. Friction causes the flame front to expand, thereby intensifying combustion and leading to acceleration as can be shown in Fig 2.2. Later, the idea was developed into theory by Bychkov et al. [15] for an exponential acceleration mechanism. The authors of [15] have found the acceleration rate, the flame shape and the velocity profiles in the flow pushed by flames for 2D channels. Akkerman et al. [17] have extended these formulations to axisymmetric, cylindrical tubes. This has further been used 
to account viscous heating [18] and also gas compression [19, 20]. With all these items figured out, the entire DDT scenario has been clarified up to the detonation onset.

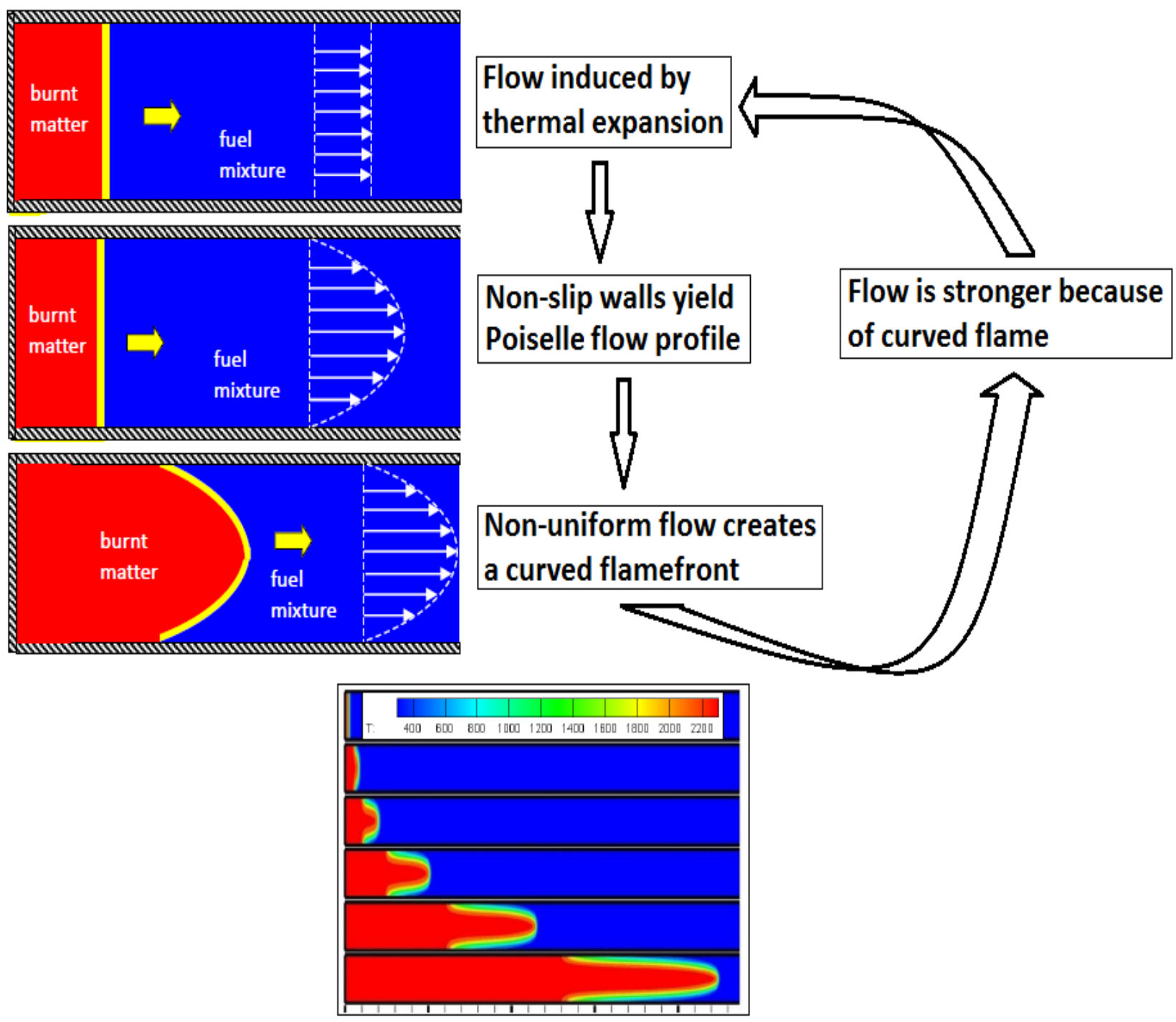

Figure 2.2: The Shelkin (wall friction) mechanism of flame acceleration $[15,16]$.

All these theories have been verified by computational simulations, while both employed some set of simplifications [15-20]. One of the assumptions employed in the theory is the Landau limit of zero flame thickness. However, later it was shown that the flame thickness influences the combustion instabilities and moderates acceleration [4,21,22]. Later, one of the flame instability mode, namely, the diffusional-thermal (DT) instability has been studied [23-26], and the domains for various instability modes have been identified. However, the simulations for non-slip, adiabatic walls [15] employed equidiffusive ( $\mathrm{Le}=1)$ flames only so far. 


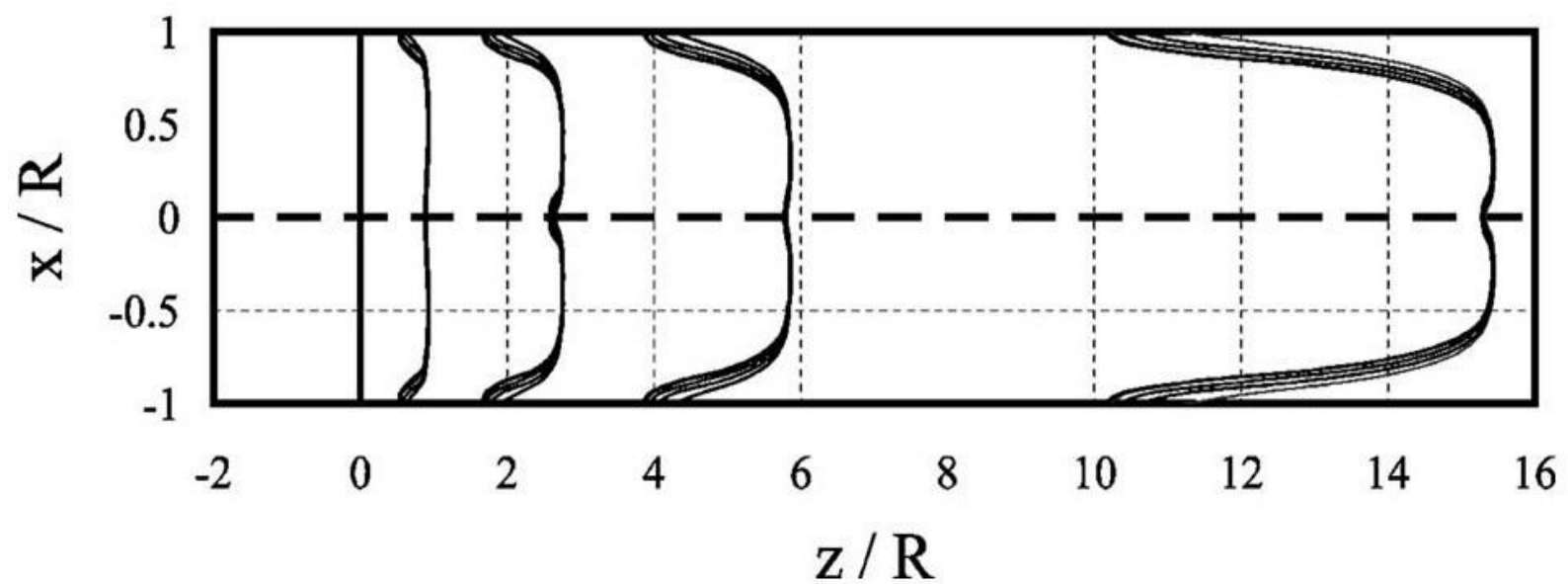

Figure 2.3: Evolution of isotherms for $\operatorname{Re}=25, \operatorname{Pr}=1$ from $600 \mathrm{~K}$ to $2100 \mathrm{~K}$ in steps of $300 \mathrm{~K}$ [15].

Even though there was reasonable progress in understanding of the flame acceleration mechanisms, there is still a lot to be explained, theoretically, that has been observed numerically. In particular, Fig. 2.3 represents the formation of little trough at the flame front near the pipe axis observed, computationally, by Bychkov et al. [15]. Darrieus [27] and Landau [28] investigated such flame perturbations in $1930-40^{\text {th }}$ and explained it to be the instability caused by the thermal expansion in the burning process. This is nowadays referred as Darrieus-Landau (DL) instability. The studies $[27,28]$ concluded that the flame corrugations rise exponentially, simultaneously increasing perturbation in the flame front, thereby enlarging the surface area due to acceleration. It was also suggested that any premixed flame is unstable (within the Landau limit of zero flame thickness).

Pelce and Clavin [29] have studied the DL instability of flames of finite thickness and explained that the heat fluxes within the flame front converge to the concave region thereby providing acceleration which stabilizes the DL instability. In non-equidiffusive flames, the DT instability effect is more profound and gains dominance over the DL instability.

The critical threshold for the DT instability can be defined in the $M k_{C}-Z e_{C}-L e_{C}-E_{A, C}$ parametric space. Here, $M k_{C}$ is the critical Markstein number, $Z e_{C}$ the critical Zeldovich number, $L e_{C}$ the critical Le number and $E_{A, C}$ the critical activation energy. The Zeldovich number (Ze) is the temperature sensitivity to the reaction rate (Eq 14). The Markstein number $(M k)$ gives the thermo-diffusive stretch effects in flame acceleration [30,31], specifically, it can be defined as the ratio of Markstein length to the laminar flame thickness: 


$$
M k=L_{m} / L_{f}
$$

where the Markstein length $L_{m}$ is the effective flame thickness with respect to the flame curvature and stretch such that the greater the Markstein length, the greater is the effect of curvature on burning velocity. The Markstein number shows the flame response to curvature and stretch and is coupled to the Lewis number [32,33,34]. The detailed formulations for these parameters are provided in Sec. 4.1.

The internal flame structure is essentially interrelated to the diffusive properties. The limits of the stability of this flame can be defined in the $Z e-L e-\Theta$ parametric space. Mostly all these are calculated and simulated in adiabatic environments as most of the effects due to heat transfer can be avoided and the concentration can be focused on the momentum and energy transfer. But the wall heat transfer processes can provide a significant impact on the flame acceleration scenario.

Hackert et al. [35] studied the laminar flames in cylindrical ducts with the heat losses at the walls, and they have shown that the solid-body radiation has a significant effect on the temperature distributions and the flame propagation speeds. Daou and Matalon [36] have also examined premixed flames in 2D channels considering the conductive heat losses at the walls.

Later, Norton and Vlachos [37] adopted thermal conductivity with variable wall thickness for a $2 \mathrm{D}$ elliptical micro-burner and have found that the wall conductivity and thickness are very important for the flame evolution as they determine the upstream heat transfer, which affects the flame ignition and stability.

Kagan et al. [38] studied deflagration-to-detonation transitions (DDT) in 2D channels with isothermal walls. All these studies have shown a significant difference as compared to the adiabatic walls. Hence the study of flame acceleration in isothermal conditions is also important. Ugarte et al. [39] has studied the effect of isothermal walls on the equidiffusive ( $\mathrm{Le}=1)$ flames.

A lot of experimental studies were also performed to analyze the flame morphology. Some of them used the setup shown in Fig 2.4 [40] to capture the shape of the flame front at different times to study the flame behavior. This helped to compare the computational data to the theory and the experimental results, to further validate the studies and understand the phenomena. 


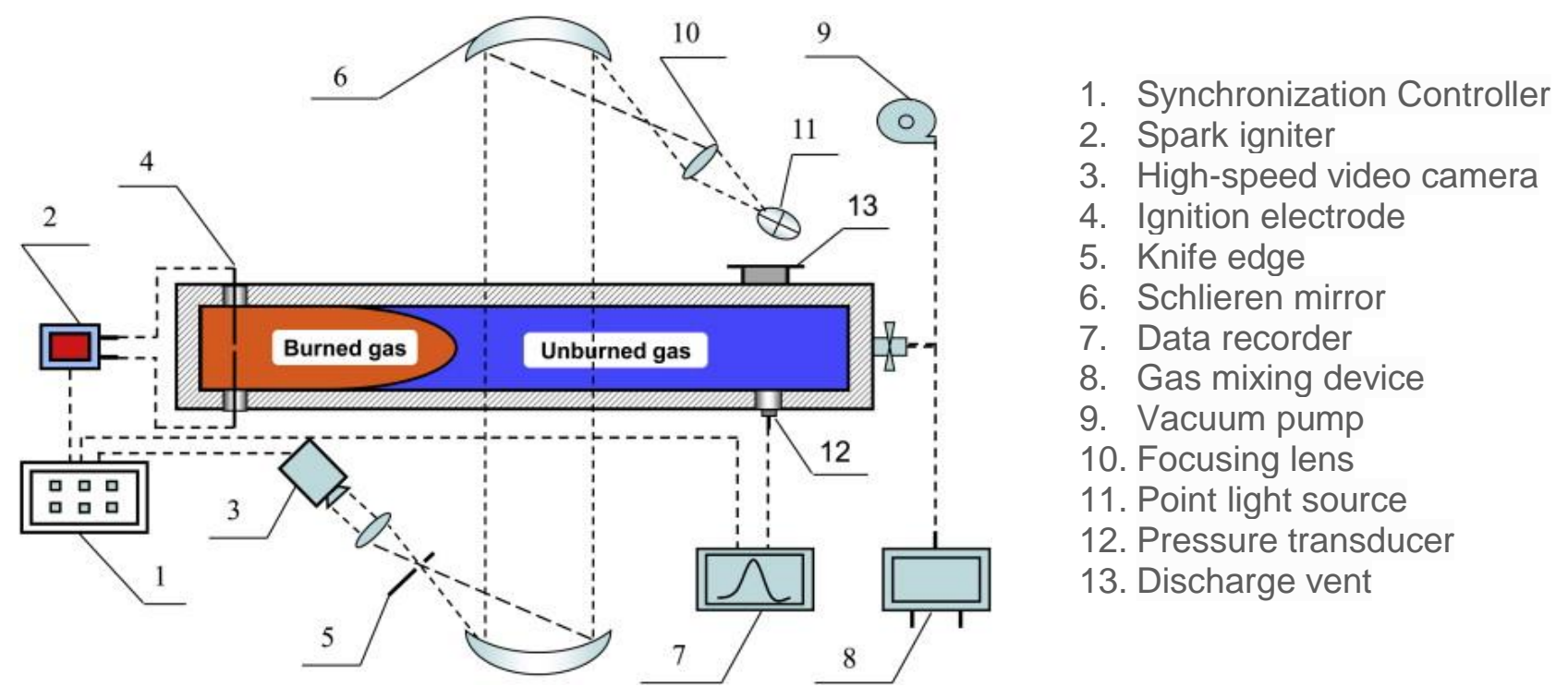

Figure 2.4: Experimental setup to capture the propagating flame front [40].

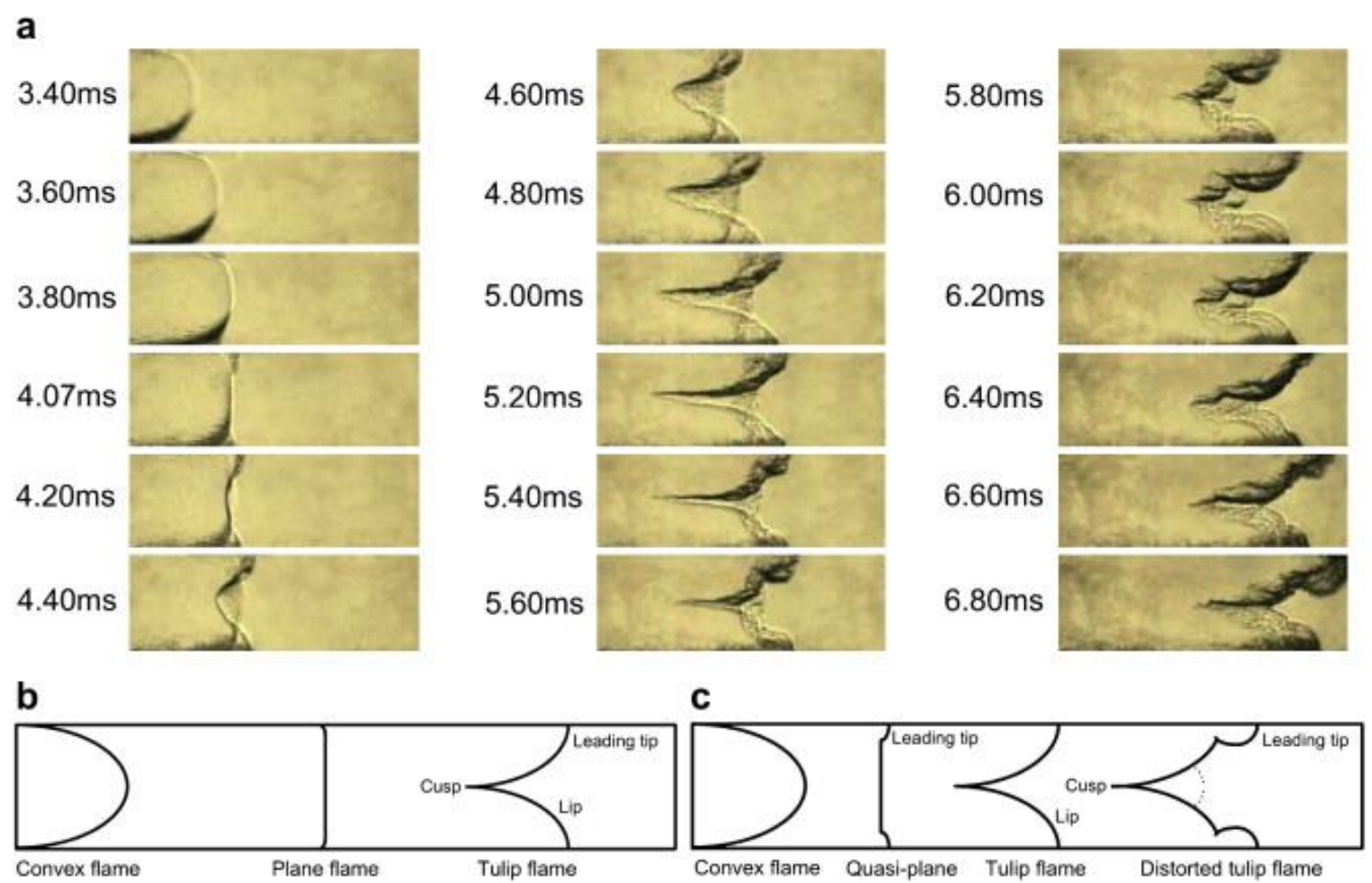

Figure 2.5: a) The images of premixed hydrogen-air flame; b) the tulip flame formation and distortion [40]

Some of the experimental images of premixed hydrogen-air flame propagation are shown in Fig 2.5. The formation of tulip flame and distortion of it, observed experimentally, is also shown. 


\section{Chapter 3: Methodology}

In the present analysis, the role of the thermal boundary conditions is explored, computationally, for $2 \mathrm{D}$ channels of various widths, having non-slip walls and one end open. Combustion is set to start at the closed end such that a flame propagates towards the open end. Numerical simulations, based on the finite-volume computational scheme, are performed. The simulations are followed by plotting and analyzing the flame morphology by scrutinizing the burning rate, flame velocity and its surface area. The evolution of the flame shape is also visualized.

The most useful parameters used to characterize the flame are the global Lewis number, which is devoted to the diffusivity, and the Reynolds number associated with flame propagation

$$
R e=\frac{R S_{L}}{v}=\frac{R}{P r L_{f}}
$$

Being the tube radius times the laminar burning velocity over the kinematic viscosity, the latter is also coupled to the scaled tube half-width $R / L_{f}$ (the Peclet, $P e$ number) and the Prandtl $(P r)$ number. With the change in Re, the stretching of the flame is affected. This can be measured using the Markstein $(M k)$ number. The model to incorporate the classical stretch formulation is adopted from the classical theory of Matalon and Matkowsky [30]. Hence, in this analysis we compare different combinations of both the Lewis and Reynolds numbers as both quantities have a great impact on the flame morphology and propagation. In this section the numerical approach used, the theories behind the boundary conditions and the grid used are explained in detail.

\subsection{Numerical Approach}

The current analysis has been performed using an in-house solver that employs finite-volume, fully-compressible numerical simulations of the set of mass, momentum and energy equations with a self-adaptive structured grid. The chemical kinetics is modelled by a one-step irreversible Arrhenius reaction of the $1^{\text {st }}$ order, with the gases assumed to obey the ideal gas law. The code is available in both Cartesian and cylindrical coordinates, and is adopted for parallel computations.

The embryo of the solver was first developed by Dr. Eriksson from Goteborg, Sweden at Volvo Aero Co., and later revised and updated by many research teams including the groups of Drs. Liberman (Uppsala University), Bychkov (Umea University), Valiev (Tsinghua University) and Akkerman (West Virginia University). Because of these edits the present analysis is possible. 
Table 3.1: Basic Equations

\begin{tabular}{|c|c|c|}
\hline Equation & Formulation & $\begin{array}{c}\text { Eq. } \\
\text { No. }\end{array}$ \\
\hline Continuity & $\frac{\partial \rho}{\partial t}+\frac{\partial}{\partial x_{i}}\left(\rho u_{i}\right)=0$ & (5) \\
\hline Momentum & $\frac{\partial}{\partial t}\left(\rho u_{i}\right)+\frac{\partial}{\partial x_{i}}\left(\rho u_{i} u_{j}+p \delta_{i j}-\gamma_{i j}\right)=0$ & $(6)$ \\
\hline Energy & $\frac{\partial}{\partial t}\left(\rho \varepsilon+\frac{1}{2} \rho u_{i} u_{j}\right)+\frac{\partial}{\partial x_{i}}\left(\rho u_{j} H+\frac{1}{2} \rho u_{i} u_{i} u_{j}+q_{j}-u_{i} \gamma_{i j}\right)=0$ & (7) \\
\hline $\begin{array}{c}\text { Species } \\
\text { conservation }\end{array}$ & $\frac{\partial}{\partial t}(\rho Y)+\frac{\partial}{\partial x_{i}}\left(\rho u_{i} Y-\frac{\mu}{S_{c}} \frac{\partial Y}{\partial x_{i}}\right)=-\frac{\rho Y}{\tau_{R}} \exp \left(-E_{A} / R_{u} T\right)$ & (8) \\
\hline $\begin{array}{c}\text { Internal Energy } \\
\varepsilon=Q Y+C_{V} T\end{array}$ & (10) \\
\hline Enthalpy & $H=Q Y+C_{P} T$ & (11) \\
\hline $\begin{array}{c}\text { Stress Tensor } \\
\gamma_{i j}=\zeta\left(\frac{\partial u_{i}}{\partial x_{j}}+\frac{\partial u_{j}}{\partial x_{i}}-\frac{2}{3} \frac{\partial u_{k}}{\partial x_{k}} \delta_{i j}\right)\end{array}$ \\
\hline $\begin{array}{c}\text { Energy } \\
\text { Diffusion } \\
\text { Vector }\end{array}$ & $q_{j}=-\zeta\left(\frac{C_{P}}{P r} \frac{\partial T}{\partial x_{j}}+\frac{Q}{S c} \frac{\partial Y}{\partial x_{j}}\right.$ & $(12)$ \\
\hline
\end{tabular}

The basic equations are presented in Table 3.1 above. The solver is parameterized with some values for a premixed combustible diatomic perfect gas for calculations, with the specifications:

- $m=2.9 \times 10^{-2} \mathrm{~kg} / \mathrm{mol}$

- $C_{V}=5 R u / 2 m$

- $C_{P}=7 R u / 2 m$

- $R_{u}=8.314 \mathrm{~J} /($ mol.K)

- $P=\rho R u T / m$ (ideal gas equation of state)

- $U_{f}=34.7 \mathrm{~cm} / \mathrm{s}$

- $\zeta=1.7 \times 10^{-5} \mathrm{~N} . \mathrm{s} / \mathrm{m}^{2}$

- $E_{A}=32 R u T_{f}$

- $M a=10^{-3}$

- $L_{f}=v / \operatorname{Pr}_{f}$

- $\Theta=5$ 


\subsection{Boundary Conditions}

Different boundary conditions are used in the simulations to implement different configurations. The conditions imposed in the analyses are:

- The non-slip wall condition is defined by $\hat{u}=0$.

- The slip wall condition is defined by $\hat{n} . \hat{u}=0$ ( $\hat{n}$ is the unit vector normal to the wall).

- The adiabatic wall condition is set using the equation $\hat{n} . \vec{\nabla} T=0$.

- The isothermal wall condition is set using the equation: $\frac{T_{\text {in }}+T_{\text {out }}}{2}=T_{w}$, where $T_{w}$ is the average value at mid-point from both surface boundaries. Specifically, we have used:

$\checkmark$ High temperature $T_{w}=800 \mathrm{~K}$ - preheated walls.

$\checkmark$ Low temperature $T_{w}=300 \mathrm{~K}$ - cold (room temperature) walls.

- The open end is set by: $P=P_{a m b}, \rho=\rho_{f},\left(u_{x}, u_{y}\right)=0$ and $Y=1$

- The Lewis number Le is changed by changing the Schmidt number Sc.

- The flame Reynolds number Le is changed by changing the radius of the channel.

\subsection{Grid Generated}

It is recalled that the solver employs the finite-volume numerical scheme. It is the $2^{\text {nd }}$-order accurate in time, $4^{\text {th }}$-order accurate in space for the convective terms, and $2^{\text {nd }}$-order accurate in space for the diffusive terms. Other computational details are explicitly explained in [41,42]. In the present investigation, the radius is varying from $20 L_{f}$ till $70 L_{f}$. The dynamic mesh is used in the solver to reduce the computational costs where the finer mesh is confined to the travelling flame front. The mesh is shown in Fig 3.1. The surrounding mesh is about 5\% coarser. The outer walls are not insulated to control the temperature hence the solver adopts an adiabatic approach.

The grid along the y-direction is $0.2 L_{f}$, which is sufficient to resolve the internal flame structure. The mesh size outside the fine grid grows gradually with $2 \%$ change in size of adjacent cells. The adaptive grid is applied to keep the flame in the fine zone, which moves together with the flame. On the other hand, the grid along the $x$-axis is taken to be uniform, with the cell size equivalent to $0.5 L_{f}$. Hence, the zone of large velocity gradients close to the walls was able to be resolved well. 


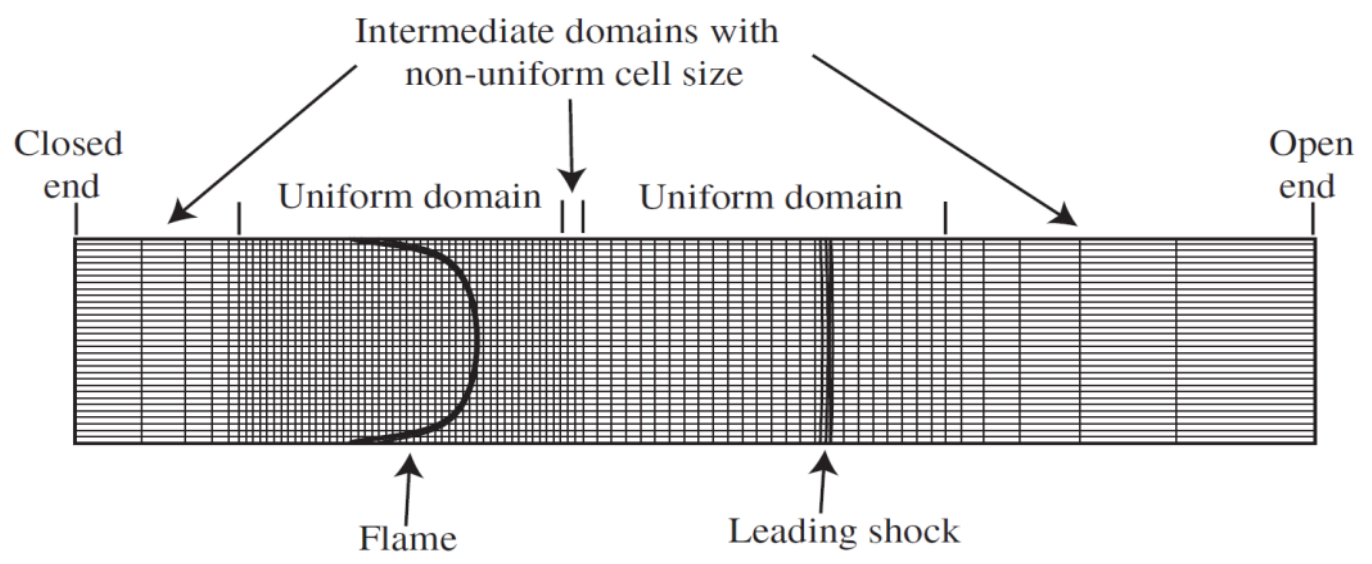

Figure 3.1: The dynamic mesh with a variable resolution [46].

To check the mesh independence, various tests simulations were run with the number of cells increased three times in $x$-direction which yielded an accuracy of (5-10)\% [21]. This can be taken as the numerical accuracy of the simulation data from Fig. 3.2.

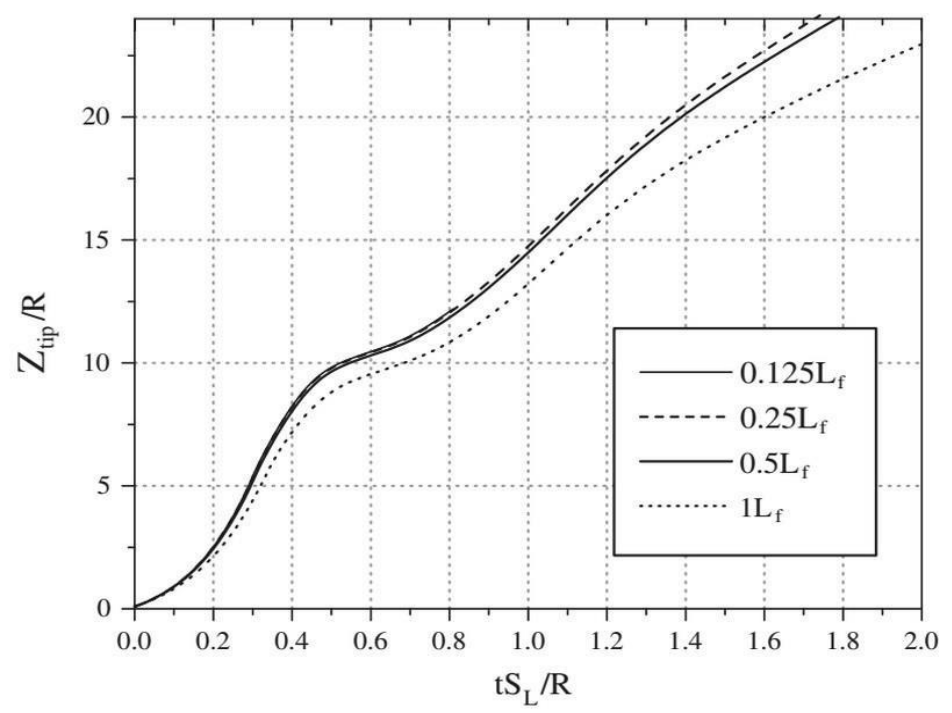

Figure 3.2: A resolution test for the flame tip position $Z_{t i p} / R$ versus the scaled time $S_{L} t / R$ for the different mesh sizes of $L_{f}, 0.5 L_{f}, 0.25 L_{f}$ and $0.125 L_{f}$ with $L e=1$ in each case [21].

We used a planar ignition to simulate all the flames considered in this study. The boundary conditions for the walls are changed as adiabatic and isothermal depending on which case we are studying. In the case of isothermal walls, the temperature of the wall is fixed to a certain value (high or low). The planar ignition as shown in Fig 3.3 follows the equations given by Zeldovich and Frank-Kamenetski. [3]. The open end is created by forcing it to be a non-reflective surface as the length of the channel has no effect on the results being studied. 


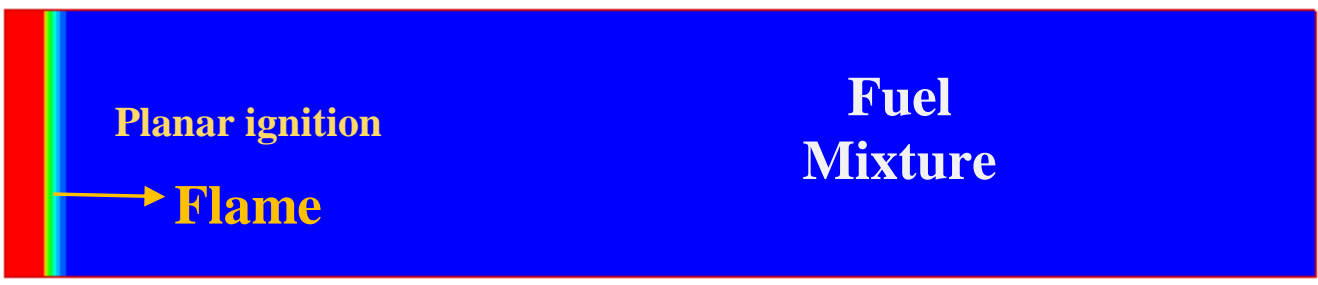

Figure 3.3: Illustration of a planar ignition.

\subsection{Validation}

The solver has been used by many researchers in Dr. Akkerman's group. To check the accuracy and validity of the solver, this section is included with the previous results produced by the solver which were compared to the experimental results to prove its precision. In 2015, a numerical simulation of stoichiometric ethylene-oxygen combustion was performed and then compared with the experiments [43]. The scaled tip velocity versus the scaled time were calculated numerically and compared to the experimental results which are shown in Fig 3.4.
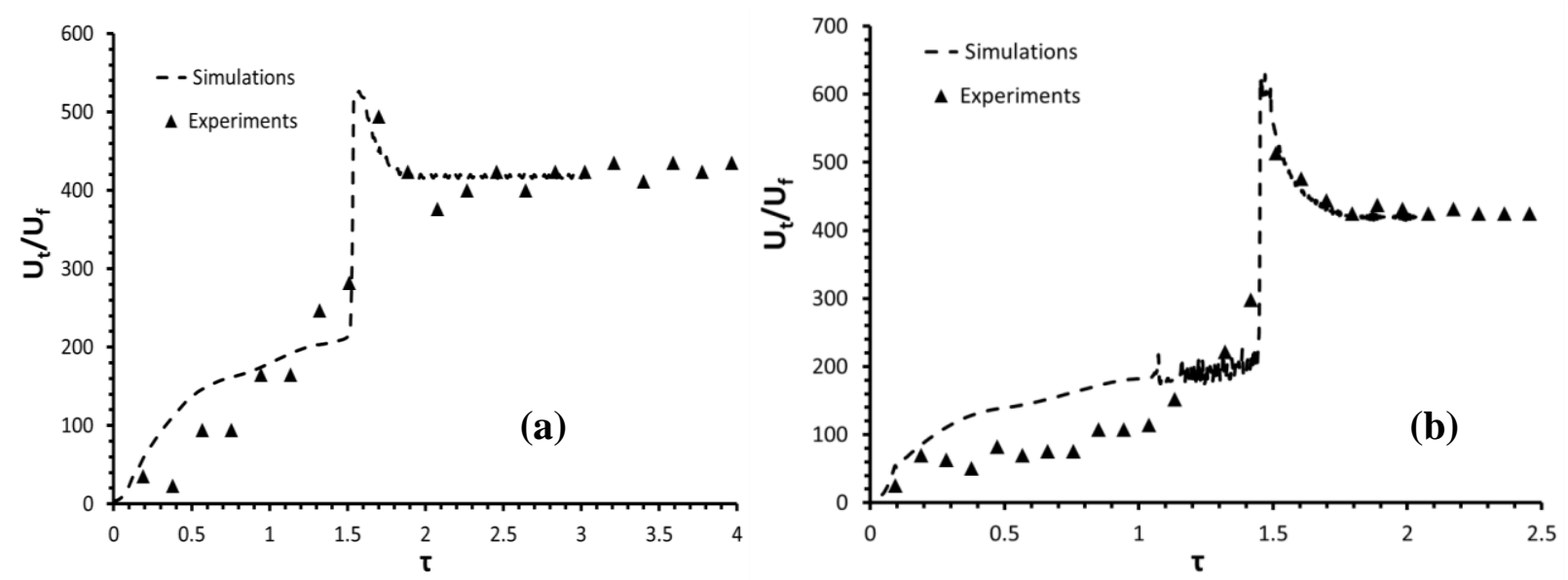

Figure 3.4: Scaled tip velocity vs the scaled time charts with both numerical and experimental results for: (a) $R=0.25 \mathrm{~mm}$; (b) $R=0.5 \mathrm{~mm}$ [43]. 


\section{Chapter 4: Theory}

There is a number of the theories which have been formulated over the time. Some of them, related to this project, are explained in this chapter.

\subsection{Zero Dimensional Theory}

The diffusional-thermal (DT) instability causes a morphological deformation of the flame front. It is recalled that the Markstein number, describing the flame response to curvature and stretch and defined as the ratio of the Markstein length to the laminar flame thickness, yields a stability limit for the DT instability. It is coupled to the Lewis and Zeldovich number as [44]

$$
M k=\frac{\Theta}{\Theta-1} \int_{1}^{\Theta} \frac{h(\vartheta)}{\vartheta} d \vartheta-\frac{1}{2} \frac{Z e(1-L e)}{\Theta-1} \int_{1}^{\Theta} \frac{h(\vartheta)}{\vartheta} \ln \left(\frac{\Theta-1}{\vartheta-1}\right) d \vartheta
$$

where, $\Theta, T_{f}$ and $\rho_{f}$ are the thermal expansion coefficient, the fuel temperature and fuel density, respectively while $T_{b}$ and $\rho_{b}$ are the parameters related to the burnt matter. The $h(\vartheta)$ is the function of temperature dependence of the transport coefficients and Ze is given by [44]:

$$
Z e=\frac{\Theta-1}{\Theta} \frac{E_{A}}{R_{u} T_{b}}=\frac{\Theta-1}{\Theta^{2}} \frac{E_{A}}{R_{u} T_{f}}
$$

Equation (13) can be simplified and written as

$$
L e=1-\frac{2 \Theta J_{1}-2(\Theta-1) M k}{Z e J_{2}}
$$

where

$$
\begin{aligned}
& J_{1}=\int_{1}^{\Theta} \frac{h(\vartheta)}{\vartheta} d \vartheta \\
& J_{2}=\int_{1}^{\Theta} \frac{h(\vartheta)}{\vartheta} \ln \left(\frac{\Theta-1}{\vartheta-1}\right) d \vartheta
\end{aligned}
$$

Fig. 4.1 represents the behavior of the $\mathrm{Ze}_{\mathrm{C}}$ as the function of $\Theta$ from Eq. (14) while Fig. 4.2 shows the change in $\mathrm{Le}_{\mathrm{C}}$ as a function of Ze from Eq. (15). After analyzing these results and further studies, it was found that the DT instability is developed only if $\mathrm{Ze}>\mathrm{Ze}_{\mathrm{C}}, \mathrm{E}_{\mathrm{A}}>\mathrm{E}_{\mathrm{A}, \mathrm{C}}$ and $L e<L e_{C}=1-Z e_{C} / Z e$, where the subscript " $C$ " indicates the critical parameters. 


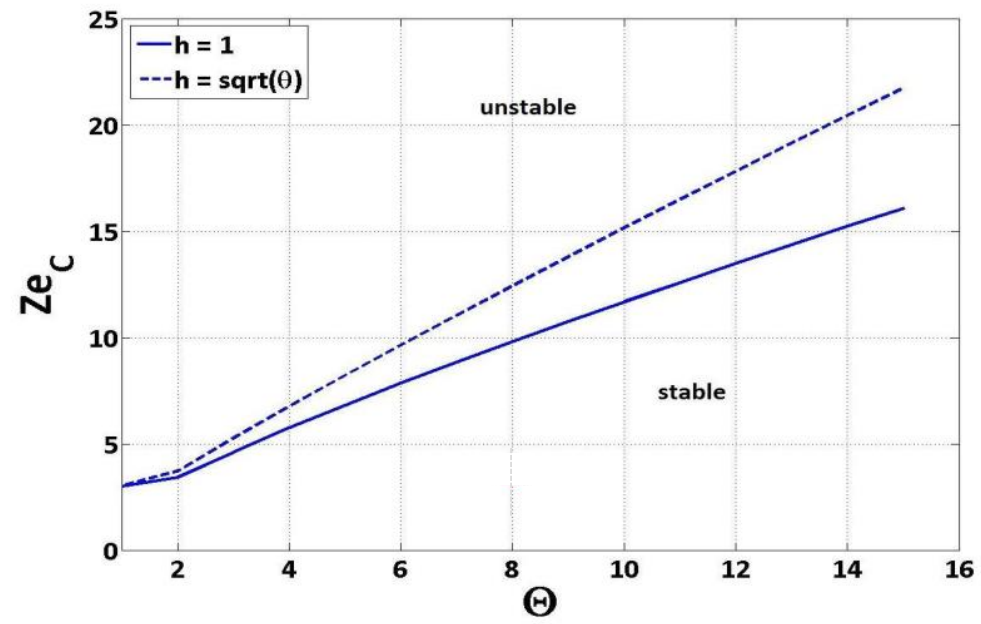

Figure 4.1: Ze $\mathrm{e}_{\mathrm{C}}$ as the function of $\Theta$ for $h(\vartheta)=1$ and $h(\vartheta)=\vartheta^{1 / 2}$.

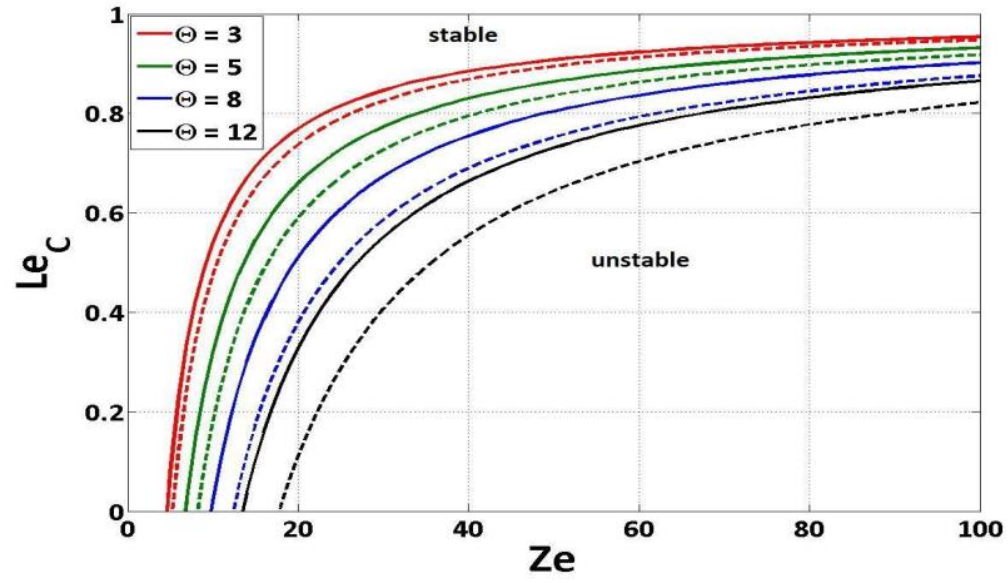

Figure 4.2: $\mathrm{Le}_{\mathrm{c}}$ as the function of Ze for $h(\vartheta)=1$ shown by the solid lines and $h(\vartheta)=\vartheta^{\frac{1}{2}}$ shown by the dotted lines.

\subsection{Theory of Flame Acceleration in 2D Channels}

All the simulations of this work are devoted to 2D channels. The respective equations describing flame acceleration are derived by Bychkov et al [15]. Specifically,

$$
\frac{\mu \cosh \mu-\sinh \mu}{\mu(\Theta-1)}=\frac{\exp \mu}{2(\mu+\sigma)}-\frac{\exp -\mu}{2(\mu+\sigma)}+\frac{\mu^{2}}{\mu^{2}-\sigma^{2}} \frac{\exp (-\sigma)}{\sigma}-\frac{1}{\sigma}
$$

where $\mu=\sqrt{\sigma R e}$. In the limit of $\mu \gg 1$, Eq. (18) reads

$$
\mu+\sigma=(\Theta-1) \frac{\mu}{\mu-1}
$$


with the analytical solution

$$
\sigma=\frac{(\operatorname{Re}-1)^{2}}{4 \operatorname{Re}}\left(\sqrt{1+\frac{4 \operatorname{Re} \Theta}{(\operatorname{Re}-1)^{2}}}-1\right)^{2} \quad \mu=\frac{\operatorname{Re}-1}{2}\left(\sqrt{1+\frac{4 \operatorname{Re} \Theta}{(\operatorname{Re}-1)^{2}}}-1\right)
$$

The simulation results of Demirgok et al. [45] explained the change in the acceleration trend depending upon the values of $\operatorname{Re}$ and $\Theta$. It was shown that acceleration is exponential for the higher values of $\Theta$ and almost linear for $\Theta$ values under the critical value. This is clearly depicted by the Fig 4.3. The circles indicate the exponential acceleration regime was tested, whereas the triangles under the critical $\Theta$ line represent the linear acceleration cases. The red stars $(\star)$ represent the simulations of the current study.

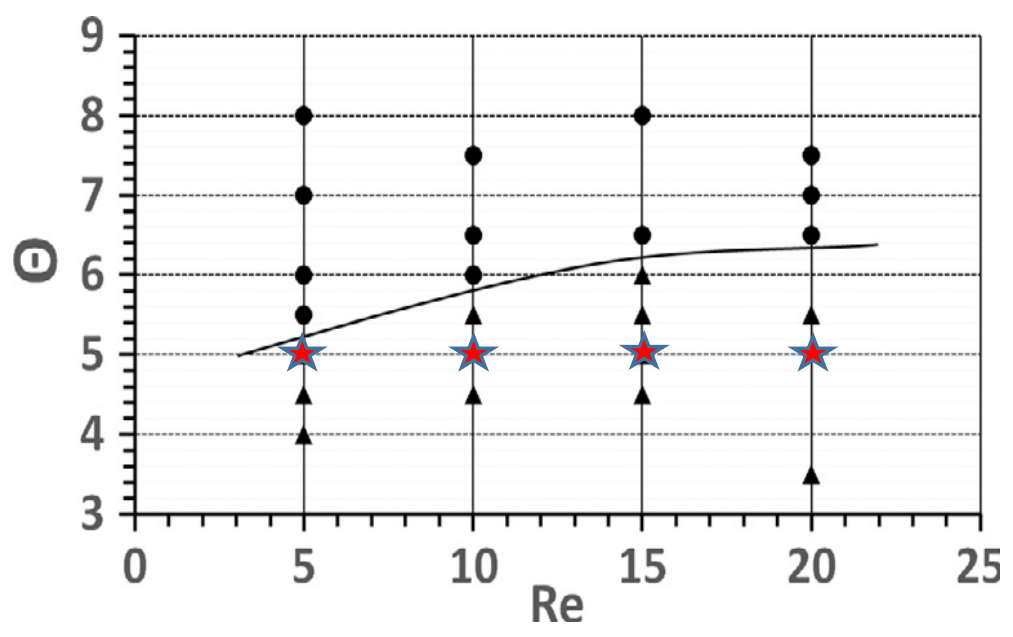

Figure 4.3: $\Theta$ vs Re diagram representing the exponential and non-exponential acceleration regimes. The solid line is the critical line where this transition takes place [45].

\subsection{Flame Acceleration in Adiabatic and Isothermal Wall Conditions}

Combustion inside a pipe is an intriguing concept. As the place for flame initiation is confined, it is largely influenced by the boundary conditions. The boundary layers formed and the heat transfers have the comparable effect on the flame dynamics. Hence, it is crucial to understand the effect of such conditions on the flame dynamics. The adiabatic case is generally considered here, because it simplifies the problem as there is no heat transfer term in it. This condition is preferred to study other factors which influence the flame morphology such as wall friction, acoustics or turbulence. Here, the non-equidiffusive flames are studied in adiabatic environment to isolate diffusivity effects without heat transfer as shown in Fig. 4.4. The simplest 
case to study the non-adiabatic condition is to consider isothermal walls as illustrated by Fig. 4.5. Here, while propagating, the flame participates in the thermal energy exchange which alters the flame structure.
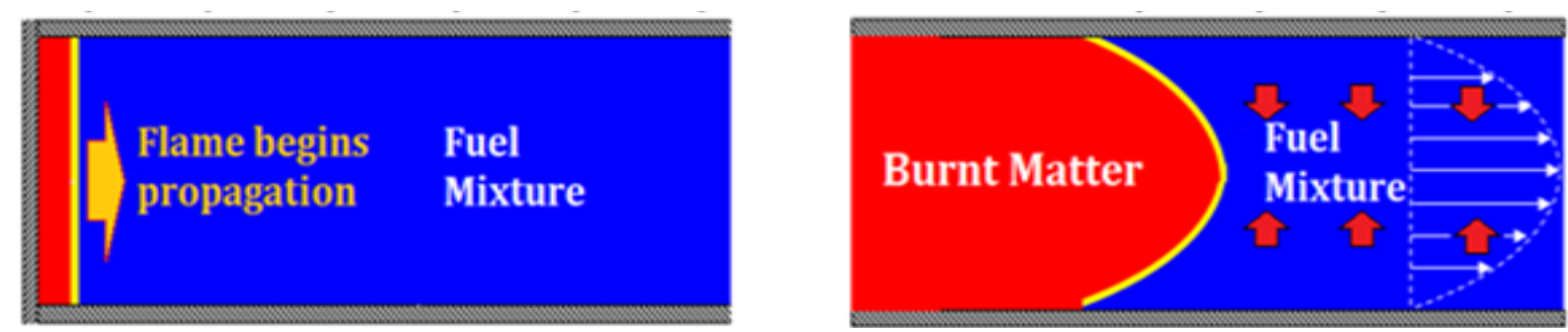

Figure 4.4: Flame structure with adiabatic walls

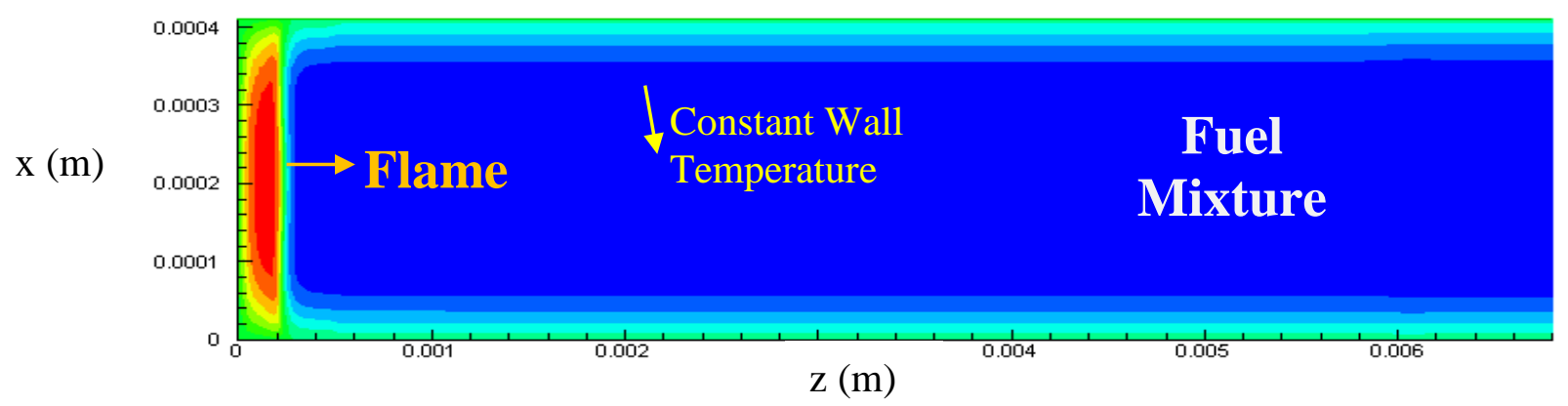

Figure 4.5: The isothermal wall conditions.

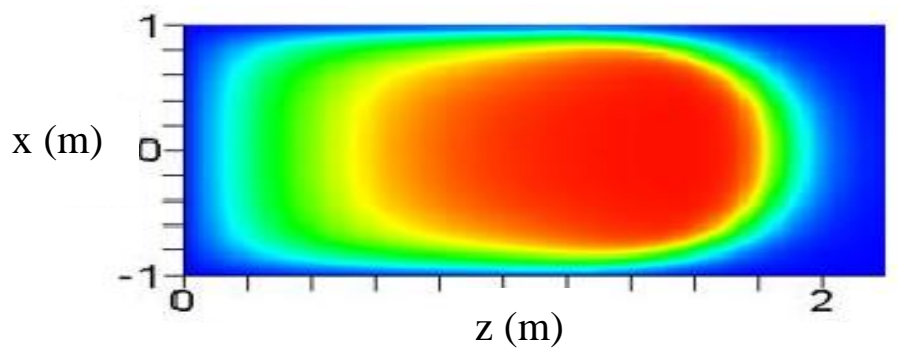

Figure 4.6: Flame structure with isothermal walls

In addition, a stimulating effect appears in the flame morphology when the channel walls are maintained at a constant temperature [39]. Namely, while at adiabatic walls flame propagation is controlled by the expanding burning matter and the flame surface area (where the fuel is actually consumed), in the case of an isothermal channel, the boundary layer causes friction influencing the flame shape, and the flame stretching is observed. It is also observed that the flame may propagate faster in isothermal conditions as the thermal expansion ratio is reduced. In the cases of isothermal walls, a different temperature gradient is observed in the flame as presented in Fig. 4.6, as the boundaries of the flame near the walls tries to gain the wall temperature. 


\section{Chapter 5: Results and Discussion}

In this chapter, the flame morphology and dynamics are analyzed in a set of parametric studies by means of computational simulations. Specifically, we consider a channel which is filled with gaseous fuel mixture. The channel has one end closed, where a planer flame is initiated and then the flame proceeds towards the open side as shown in Fig. 4.4. Two wall boundary conditions are considered: (i) adiabatic and (ii) isothermal walls, respectively. The burning matter expands thereby pushing the flame forward because of thermal expansion. Due to wall friction, the flow ahead of the flame becomes non-uniform thereby bends the flame front. As a result, the flame surface area increases, and such a corrugated flame consumes more fuel per unit time and accelerates faster than the planar one at the same thermal-chemical conditions. Consequently, a flame continuously accelerates due to the thermal and boundary conditions until the deflagrationto-detonation transition. Wall friction impacts the flame propagation by producing the flow similar to a Poiseulle flow as shown in Fig. 4.4.

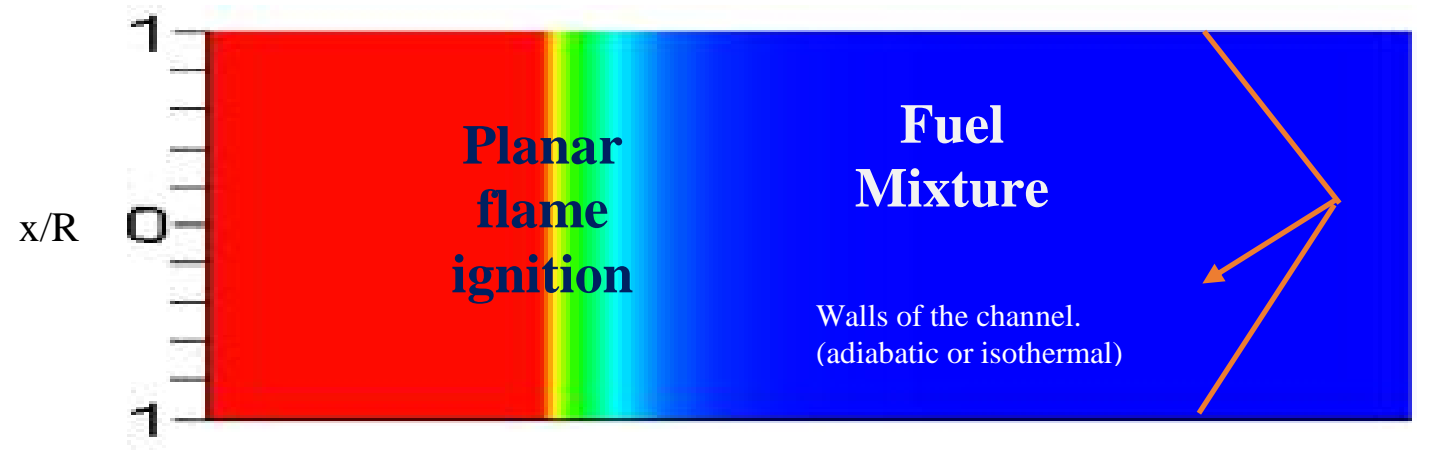

Figure 5.1: Computational thermal model created by the solver.

Figure 5.1 shows the general thermal model created by the solver to solve the considered cases. The wall conditions are set accordingly and then the flame propagates. Furthermore, the shape of the flame front as it progresses depends on several factors such as the Lewis and Reynolds numbers, wall boundary conditions etc. In the following sections, we investigate the effect of these factors by means of the detailed parametric study. While we keep one or two parameters as a constant, we investigate variation of the other parameter(s).

This section is divided into five parts, where the first subsection is devoted to equidiffusive $(\mathrm{Le}=1)$ flames in a channel with adiabatic walls; followed by the next two subsections devoted 
to the non-equidiffusive ( $\mathrm{Le}<1$ and Le $>1$ ) flames under the same wall boundary conditions with various flame Reynolds numbers. The last two sections are dedicated to the isothermal wall conditions with low $\left(T_{w}=300 \mathrm{~K}\right)$ and high $\left(T_{w}=800 \mathrm{~K}\right)$ wall temperatures. For all these analyses, we took the thermal expansion factor as $\Theta=5$ which provides a relatively low energy release in the chemical reaction as compared to $\Theta=8$, which is typical for methane and propane burning.

All these factors have a significant impact on the flame morphology and dynamics. The flame velocity calculated will be different as the local velocities are affected by these behavioral changes whereas the planar flame velocity remains the same. Consequently, the burning rate also changes, and it can be calculated as:

$$
U_{w}=\frac{1}{\rho_{f} R} \int \frac{\rho Y}{\tau_{R}} \exp \left(-E_{A} / R_{u} T\right) d x d z
$$

\subsection{Equidiffusive Flames with Adiabatic Walls}

In this subsection the equidiffusive $(\mathrm{Le}=1)$ flames are considered at various $\mathrm{Re}$ (controlled by varying the channel width). These cases are studied to create a benchmark to compare, later, the properties of the non-equidiffusive accelerating flames. The flame Reynolds number is varied in the range $5 \leq \mathrm{Re} \leq 30$. The width of the channel is therefore in the range $10 \leq \mathrm{R} \leq 60$. The graphs in Fig. 5.2 are for (a) $\operatorname{Re}=5$ and (b) $\operatorname{Re}=10$. We observe that all the properties have a smooth transition and exponential behavior. However, increasing Re further, Figs. 5.3 - 5.5, we observe that the growth of the scaled properties become not smooth anymore.

Additionally, we can observe that there are some sudden peaks in all the properties for the considered Re numbers. This might be due to the trough formation in the flame front, which is related to some artifacts due to the initial conditions, or because of the combustion instability at the channel axis [13]. As the channel size increases, we see that the trough formation becomes more evident. For higher $\operatorname{Re}, \operatorname{Re}=30$, we also see that the trough becomes prominent and the finger projections start forming. The flame front in the channels with lower Re does not show such a behavior. We can see from Figs. 5.4 and 5.5, that the increase in Re reduces the scaled flame tip velocity and the flame surface area. Additionally, a flame exhibits linear acceleration regime in the range of $15 \leq \operatorname{Re} \leq 30$, while it accelerates exponentially for the $5 \leq \operatorname{Re} \leq 10$. 
(a)

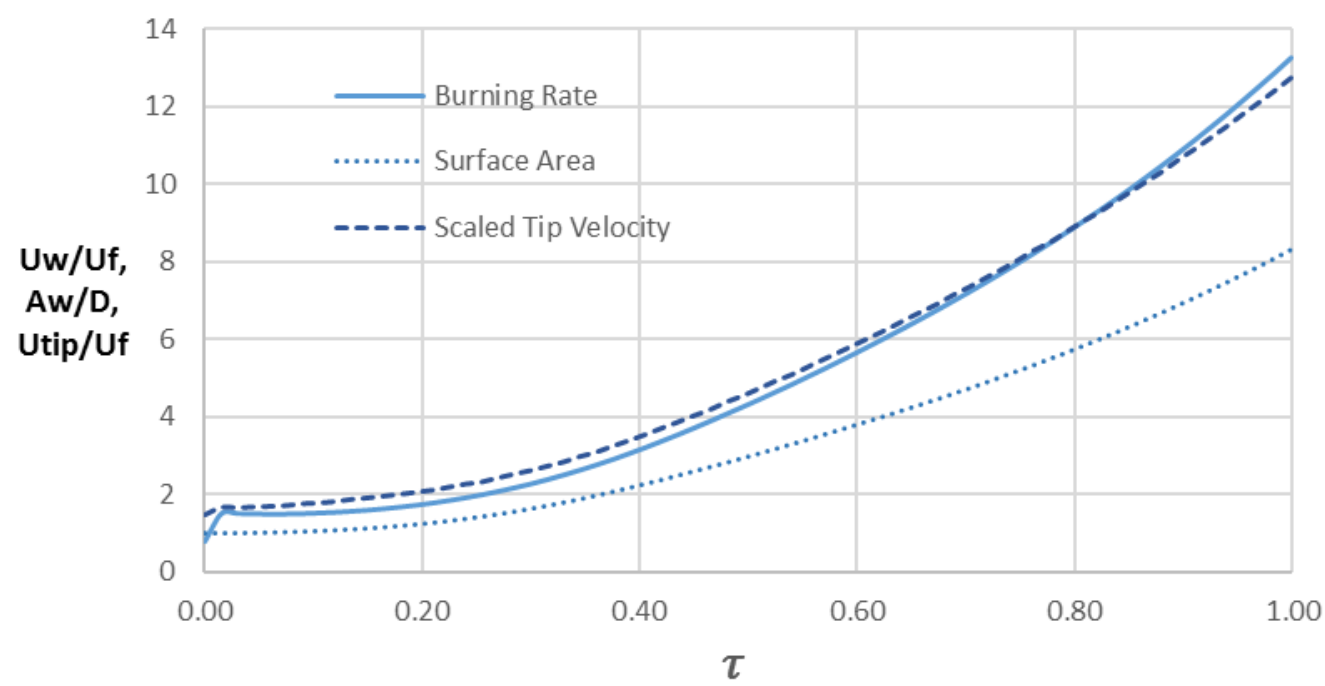

(b)

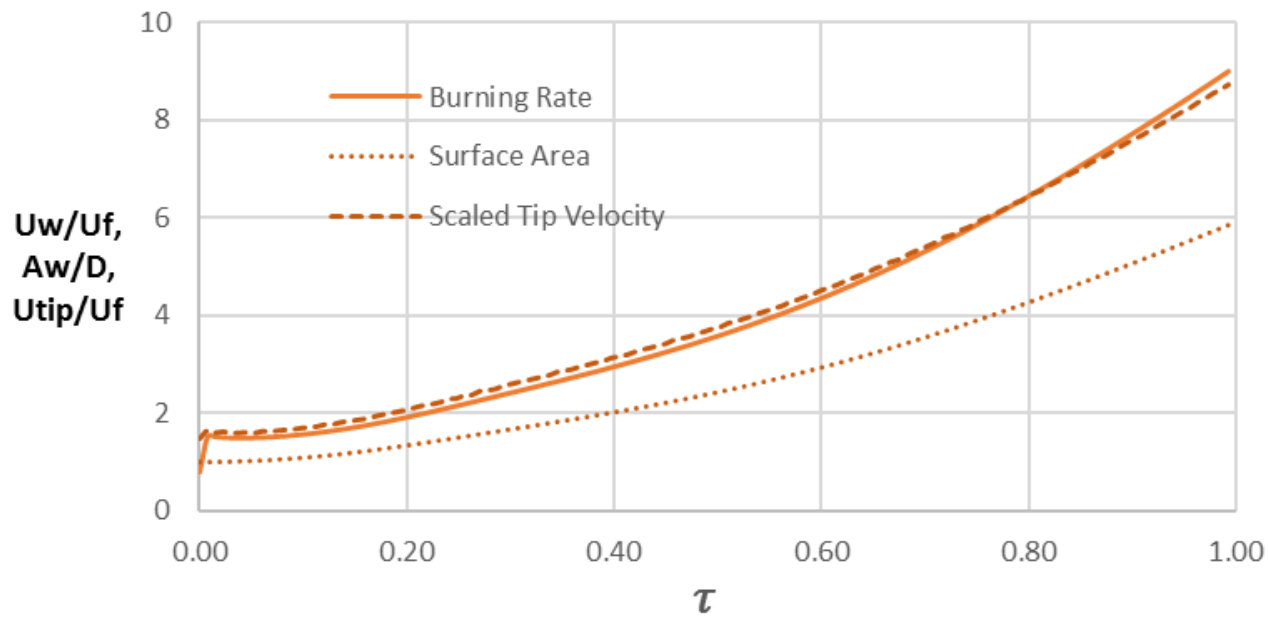

Figure 5.2: The scaled flame tip velocity, $U_{\text {tip }} / U_{f}$, the scaled surface area, $A_{w} / D$, and the scaled total burning rate, $\mathrm{U}_{\mathrm{w}} / \mathrm{U}_{\mathrm{f}}$, versus the scaled time $\tau=\mathrm{U}_{\mathrm{f}} \mathrm{t} / \mathrm{R}$ for $\mathrm{Le}=1$ and $\mathrm{Re}=5$ (a) and 10 (b). 


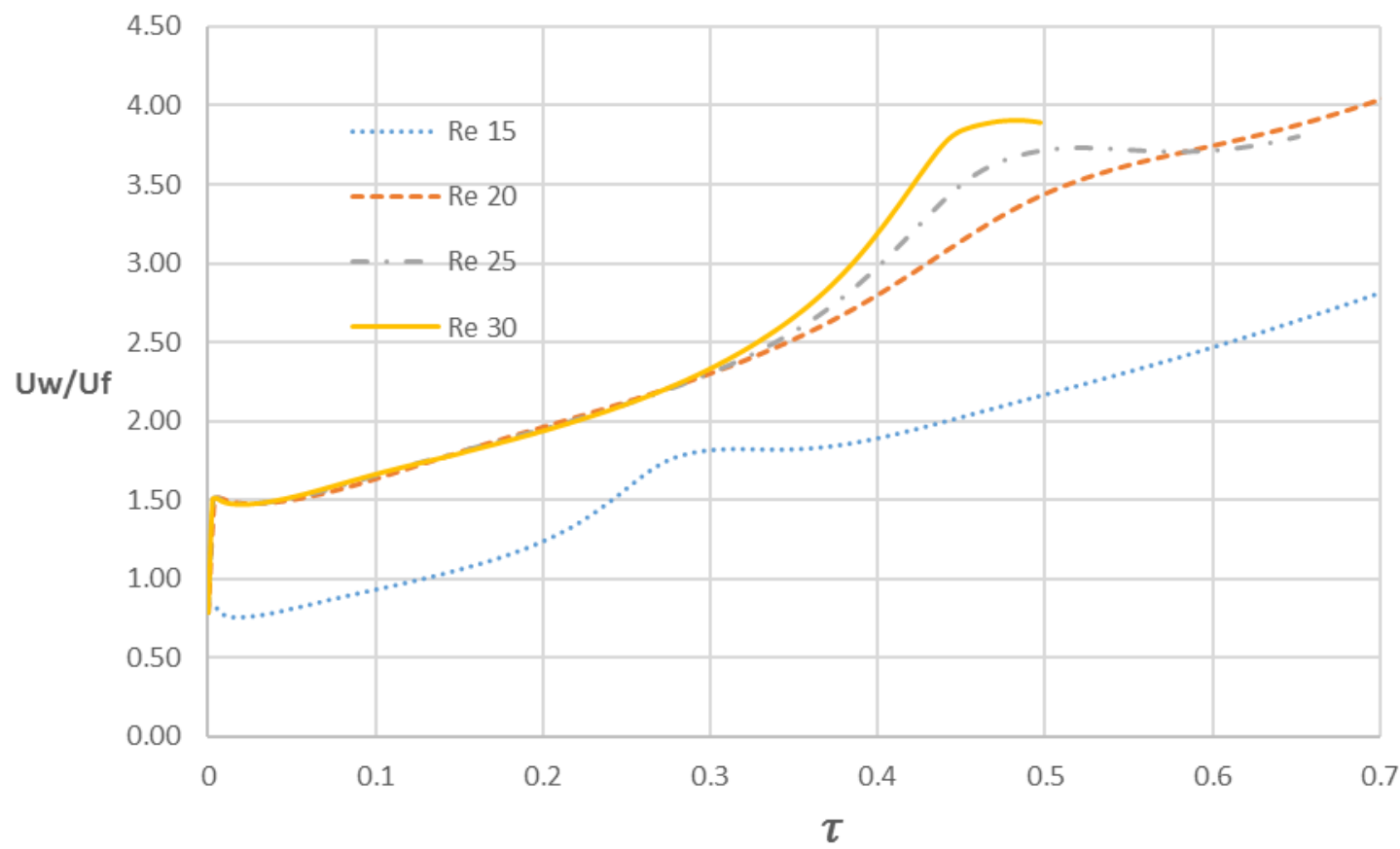

Figure 5.3: The scaled burning rate $U_{w} / U_{f}$ versus the scaled time $\tau=U_{f} t / R$ for different flame Reynolds numbers $\operatorname{Re}=15 \sim 30$.

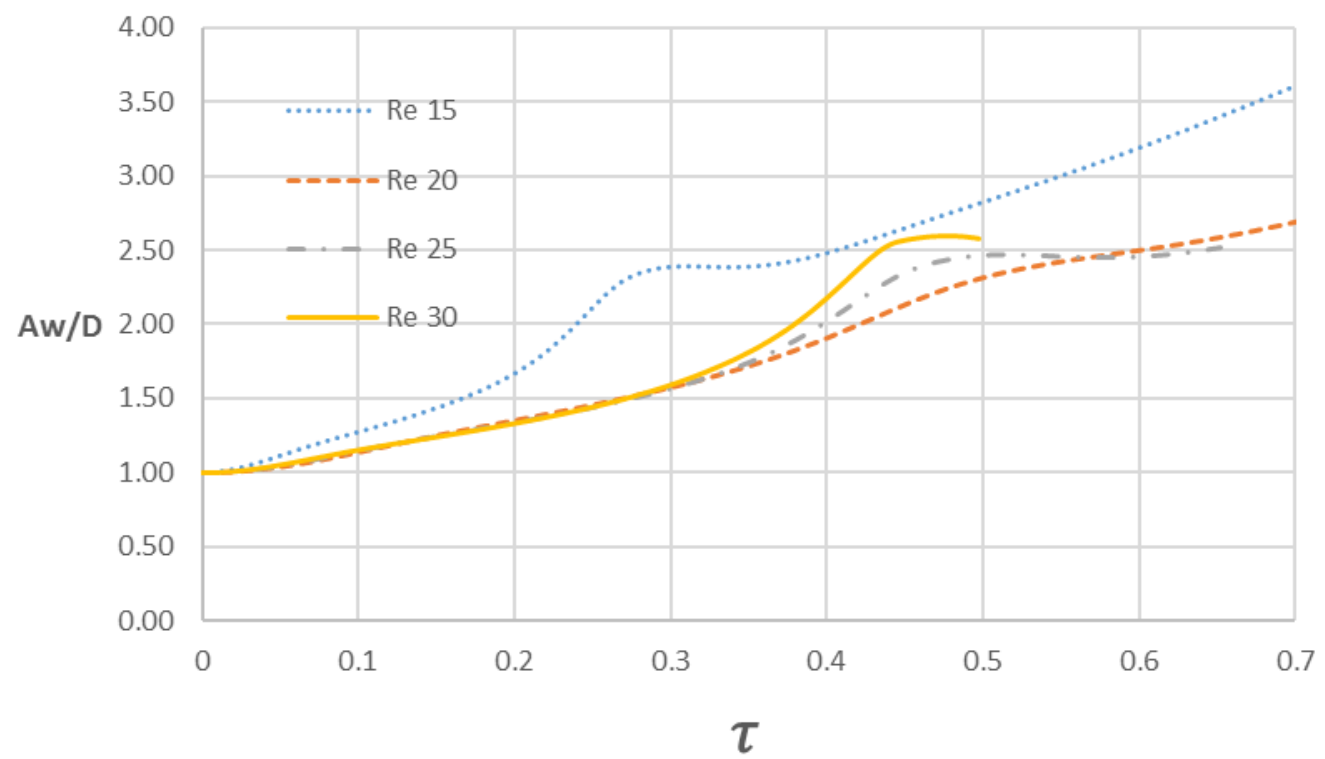

Figure 5.4: The scaled flame surface area $A_{w} / D$ versus the scaled time $\tau=U_{f} t / R$ for different $R e$. 


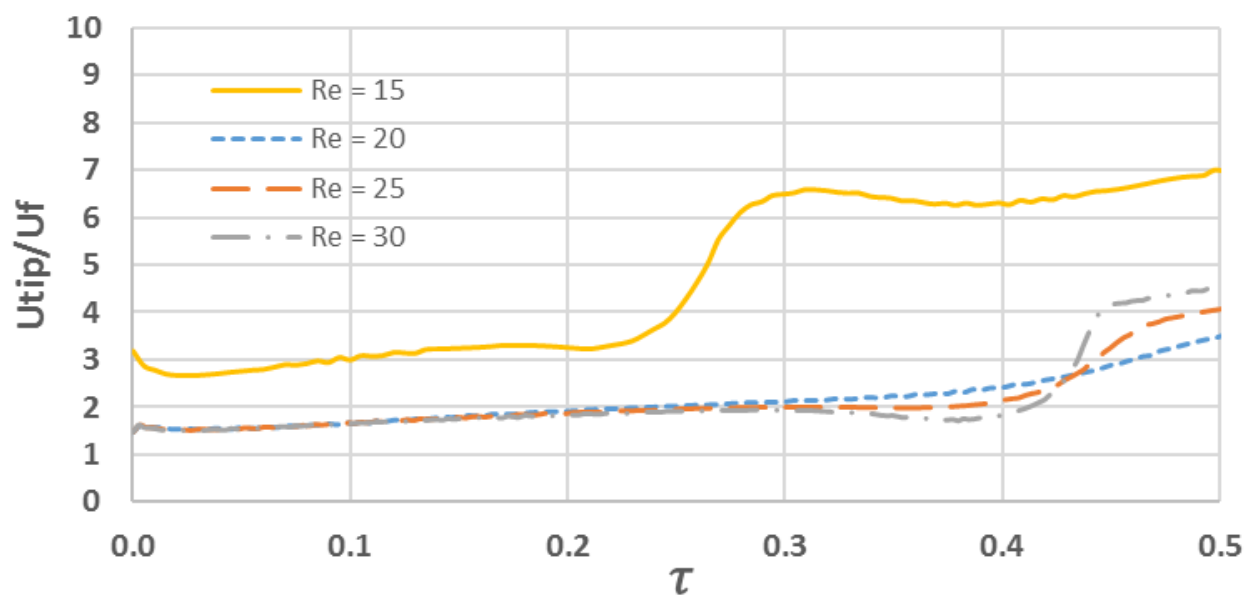

Figure 5.5: The scaled flame tip velocity $\mathrm{U}_{\mathrm{tip}} / \mathrm{U}_{\mathrm{f}}$ vs the scaled time $\tau=\mathrm{U}_{\mathrm{f}} \mathrm{t} / \mathrm{R}$ for different Re.

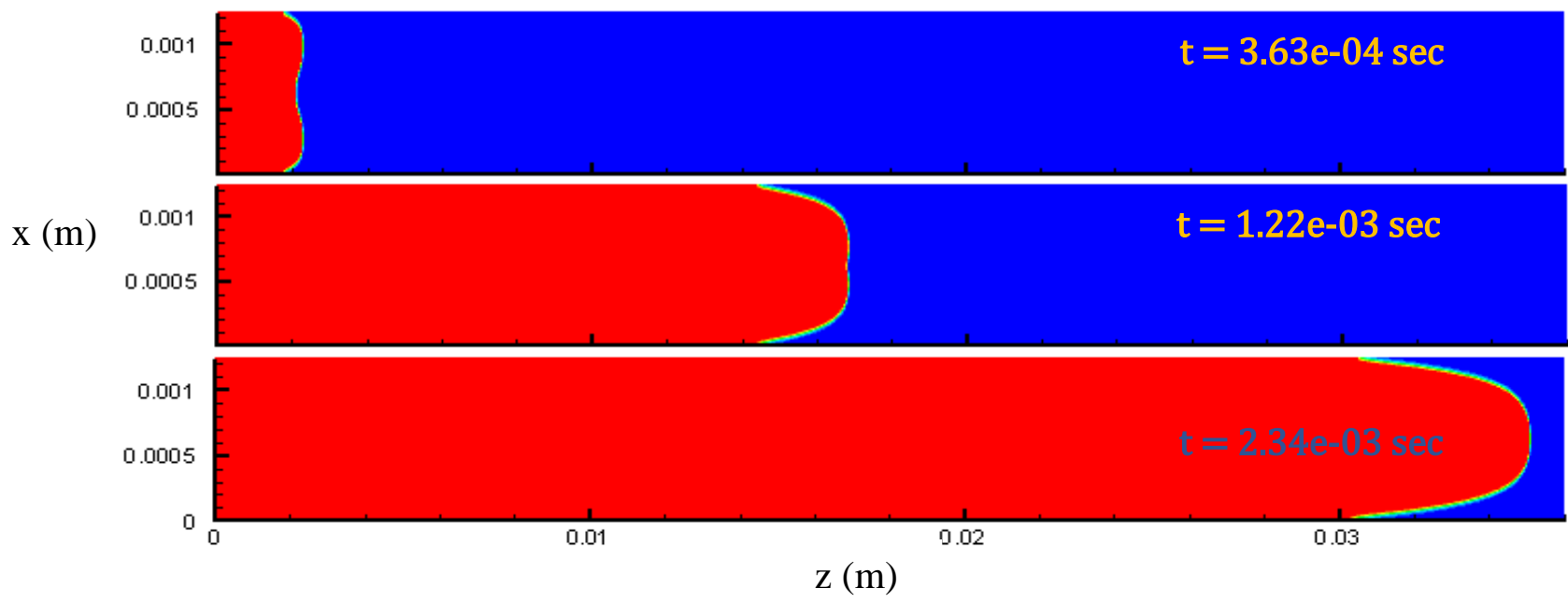

Figure 5.6: The color temperature snapshots of the flame evolution for $\mathrm{Le}=1$ and $\mathrm{Re}=15$.

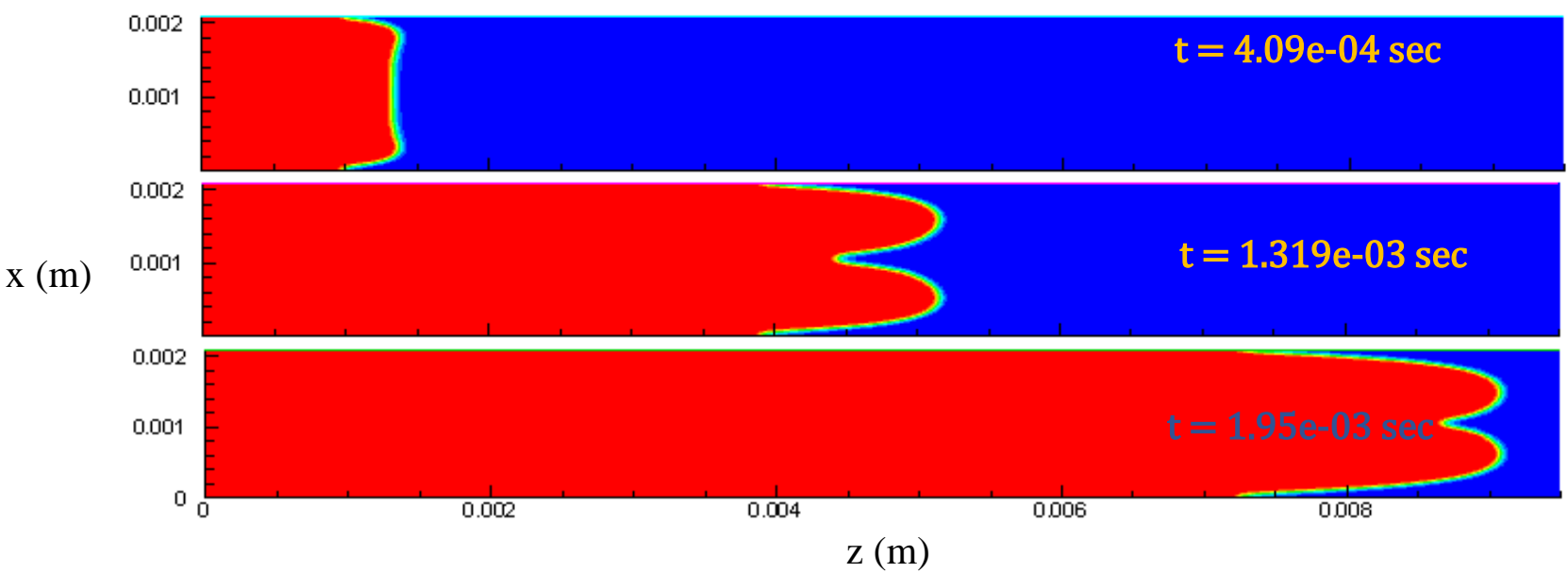

Figure 5.7: The color temperature snapshots for the flame evolution for $\mathrm{Le}=1$ and $\mathrm{Re}=25$. 
The flame evaluation is seen in Figs. 5.6 and 5.7 for $\mathrm{Re}=15$ and 25, respectively. Specifically, Fig. 5.7 shows the evidence of trough formations and birth of the finger-like flame projections. These fingers become more noticeable as the flame propagates further. As the flame area increases, due to the trough formation, the flame consumes more fuel and accelerates faster. This can be easily understood by the consecutive flame tip snapshots in Figs. 5.6 and 5.7.

\subsection{Le <1 Flames with Adiabatic Walls}

In this subsection, we study non-equidiffusive flames to understand the effects of mass and thermal diffusivity on the flame propagation and to analyze the DT instability modes with respect to the flame structure and dynamics. Specifically, $0.2 \leq$ Le $<1$ cases are examined. Referring to Figs. 5.8 and 5.9, the trough formations become more concave which in turn increases the surface area, burning rate and flame acceleration. In fact, the Le $<1$ flames exhibit an effective flame "channeling", which is the splitting of flame into two or more elongated crests with morphological deformation resembling the DT instability.

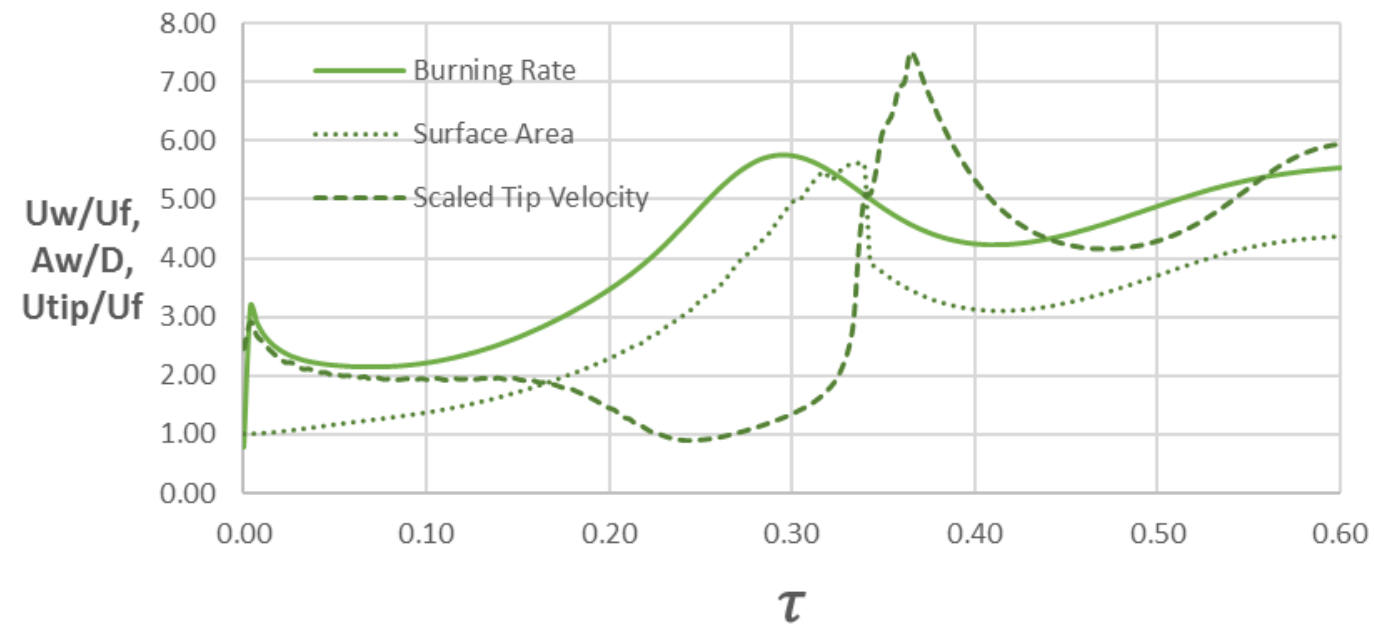

Figure 5.8: The properties of flame front versus the scaled time for $\mathrm{Le}=0.2$ and $\mathrm{Re}=20$.

Figure 5.8 shows the time evolution of the scaled burning rate, the scaled flame surface area and the flame tip velocity. As it can be seen in Fig. 5.9, trough formation heightens as Le decreases and the two crests create a split at the channel axis, and it gives rise to a third crest at the middle. As the time grows, we see an increase in the flame surface area and the burning rate, which promotes acceleration significantly. Later, due to drastic acceleration, the middle crest collapses 
and closes the gap between the two troughs. This suddenly decreases the flame surface area, which promptly moderates flame acceleration.

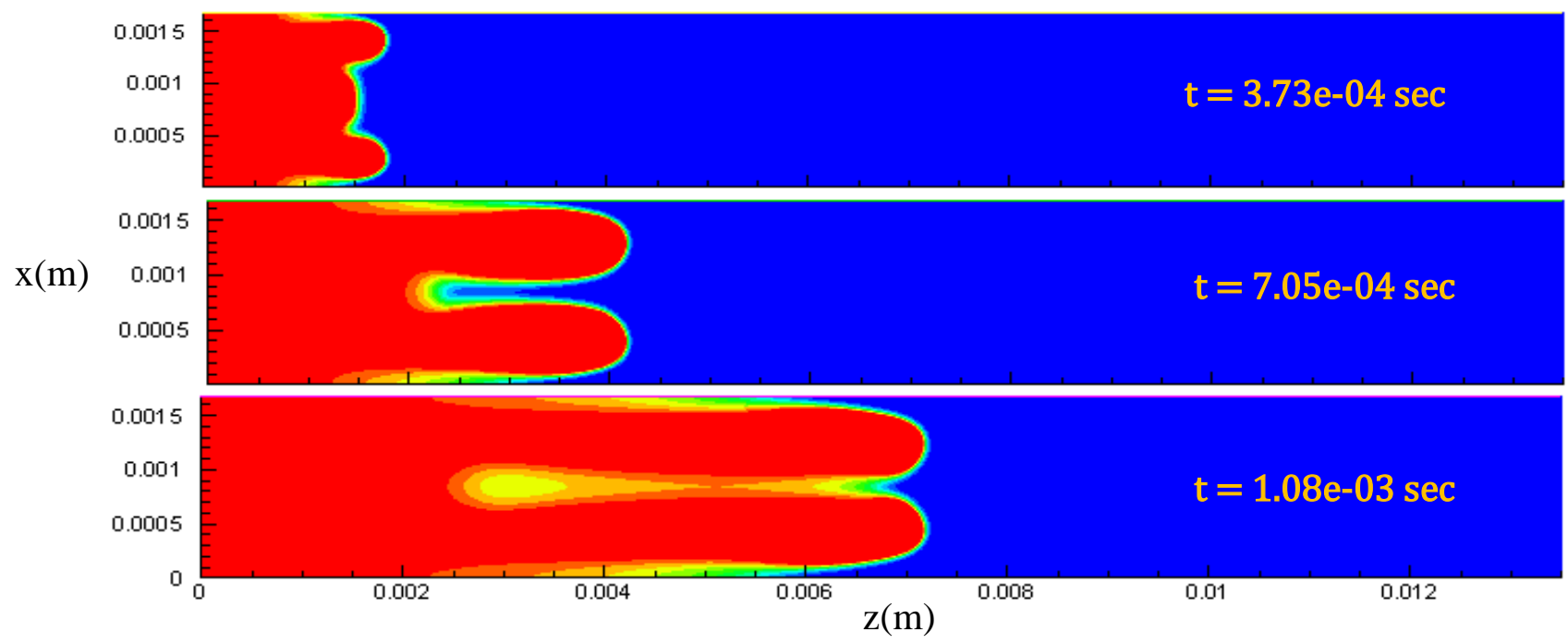

Figure 5.9: The color snapshots of the flame evolution for $\mathrm{Le}=0.2$ and $\mathrm{Re}=20$.

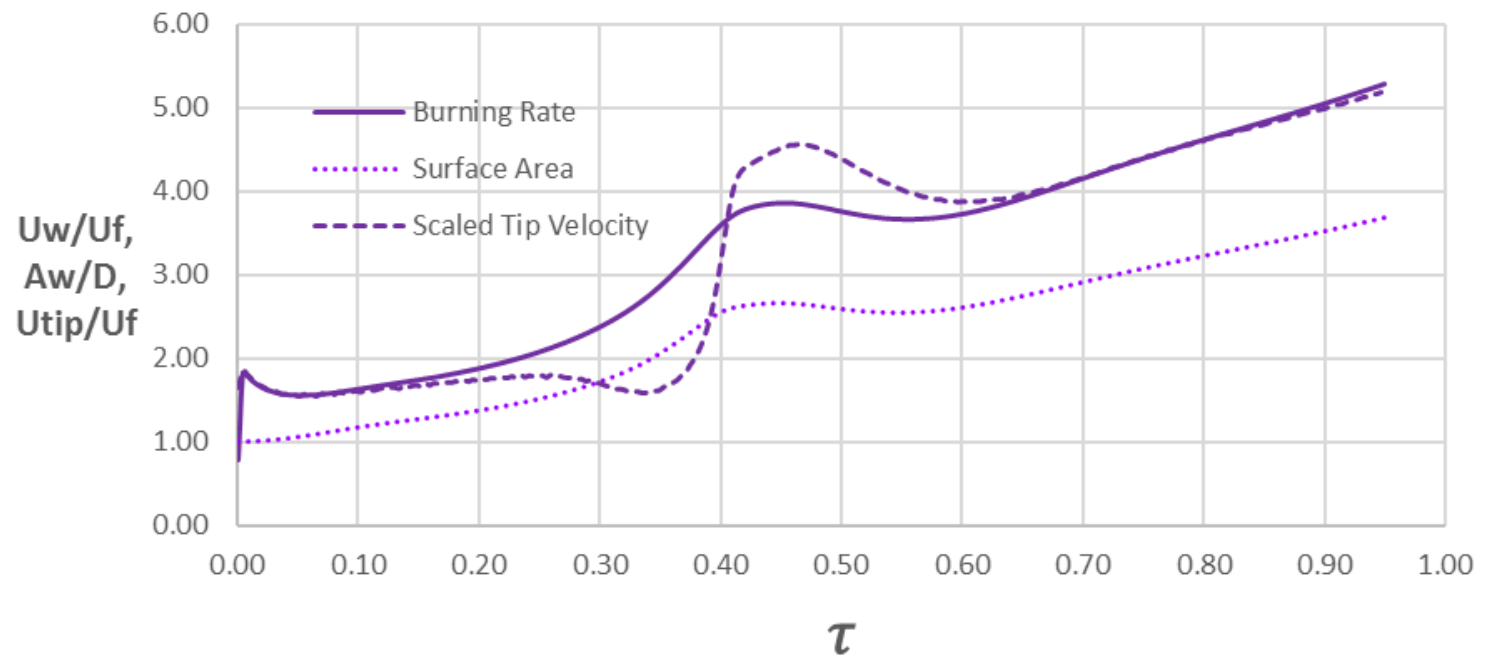

Figure 5.10: The properties of flame front versus the scaled time for $\mathrm{Le}=0.6$ and $\operatorname{Re}=20$.

As the Lewis number increases to Le $=0.6$, formation of the third trough is not as apparent as it can be seen in Fig. 5.11. Indeed, this difference can be easily judged by comparing Fig. 5.8 (for Le $=0.2$ ) to Fig. 5.10 (for Le =0.6). Hence, referring to Figs. 5.9 and 5.11, it can be concluded that flame channeling decreases with Le. Along with the change in the Lewis number, the flame propagation Reynolds number also provides a noticeable impact on the flame shape. In Fig. 5.13, we increase Re further, and the center crest becomes more noticeable. This also results 
in splitting the elongated crests, which exposes larger surface area and, thereby, the flame will accelerate even much faster.

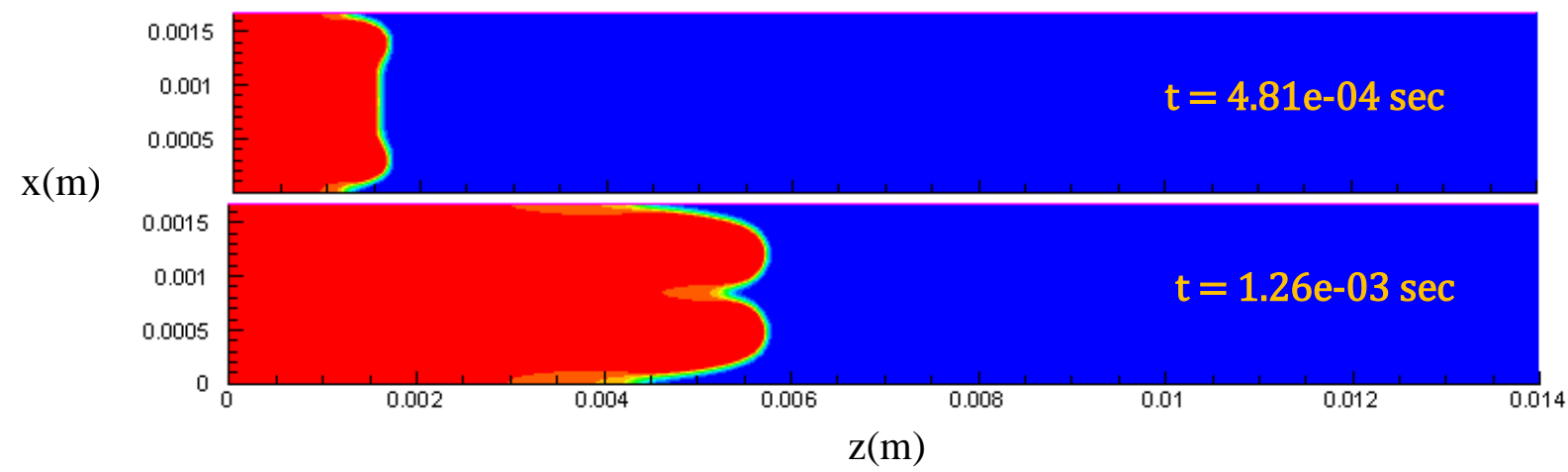

Figure 5.11: The color snapshots of the flame evolution for $\mathrm{Le}=0.6$ and $\operatorname{Re}=20$.

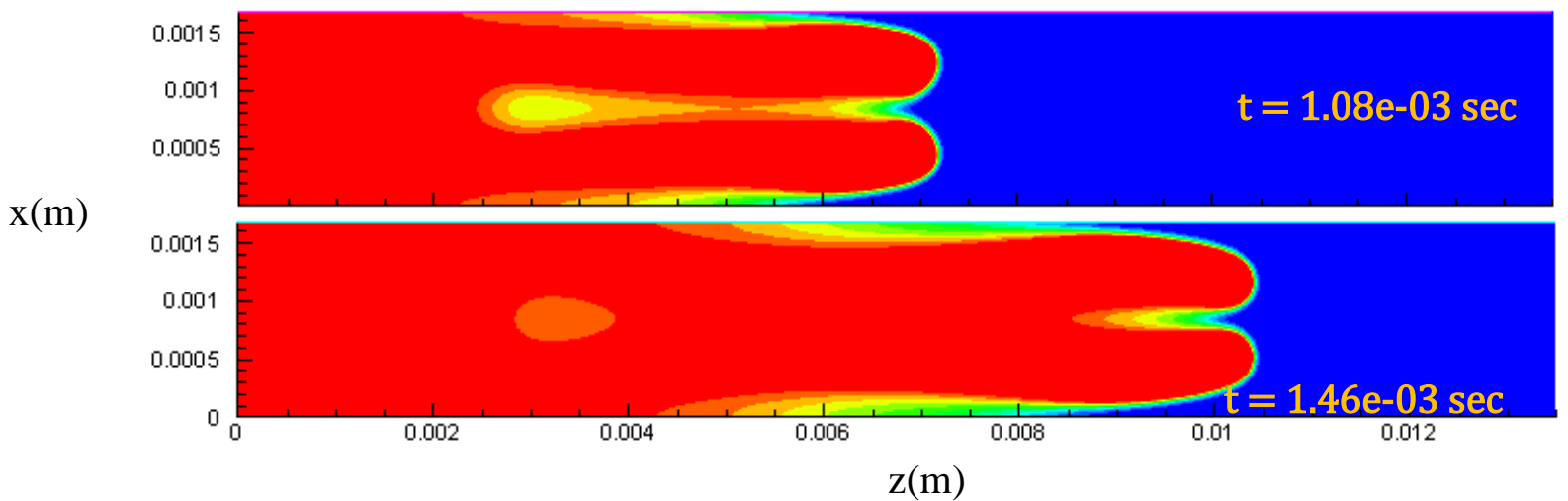

Figure 5.12: The color snapshots of the flame evolution for $\mathrm{Le}=0.2$ and $\mathrm{Re}=20$

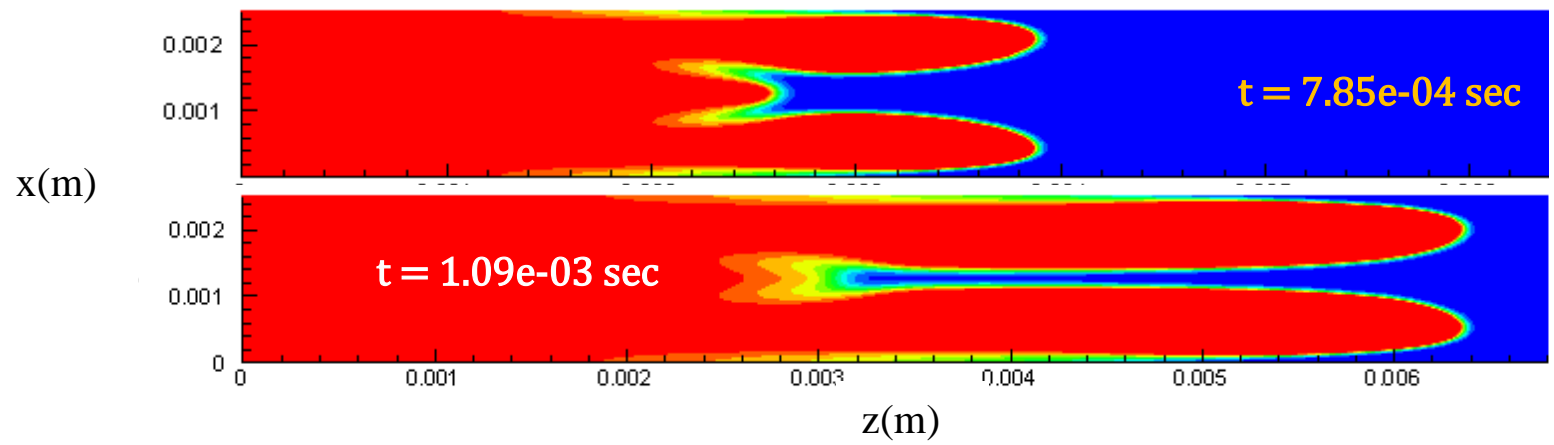

Figure 5.13: The color snapshots of the flame evolution for $\mathrm{Le}=0.2$ and $\mathrm{Re}=30$.

After examining the flame structures for all non-equidiffusive flames, the flame acceleration stages can be specified. Here, the first stage is initial trough formation, followed by the split of this formation with the elongated crests that enhance flame acceleration. This stage is succeeded by the closing of the split, i.e. the wall crests start attaching by the third crest which catches up 
with the first two crests from the center. The final stage is that when the entire flame front moves and when the axial crest combines with the upper and lower crests to create the entire flame front without any breaks [21].

\subsection{Le >1 Flames with Adiabatic Walls}

This section examines non-equidiffisive, fuel-rich (Le > 1) hydrocarbon flames. It is recalled, in this respect, that the Le exceeding unity implies a dominance of the thermal diffusivity on the mass diffusivity. Specifically, the cases of $\mathrm{Le}=1.4$ and $\mathrm{Le}=2.0$ are investigated at a fixed flame Reynolds number, $\mathrm{Re}=20$. Some curious conceptual differences can be concluded from Figs. 5.14 and 5.15 for the non-equidiffusive flames, i.e. the flame channeling behavior was observed to be relatively minor as compared to the Le $<1$ cases. Additionally, there is no sight of a center crest or a split in Figs. 5.14 and 5.15. Similar behaviors are also observed for higher Reynolds number, $\operatorname{Re}=30$, for Le $=1.4$ and Le $=2.0$ as shown in Figs. 5.16 and 5.17, respectively.

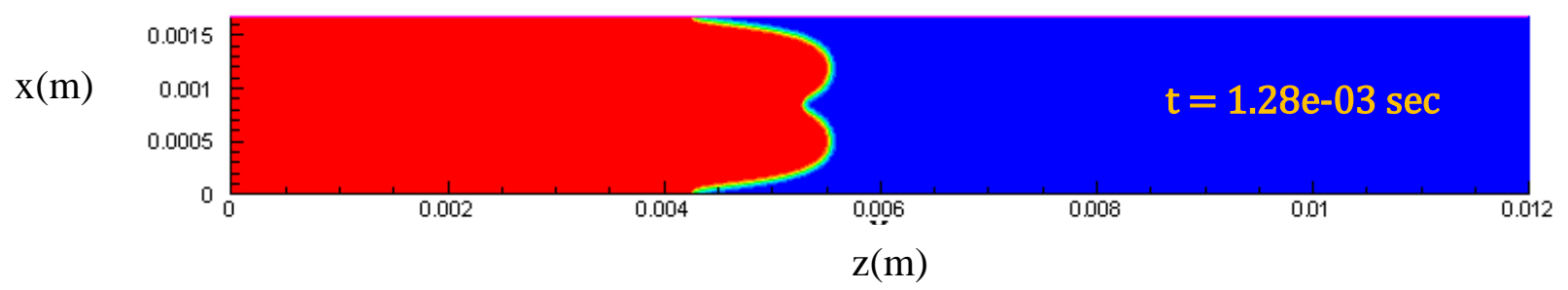

Figure 5.14: A temperature snapshot for a flame with $\mathrm{Le}=1.4$ and $\operatorname{Re}=20$.

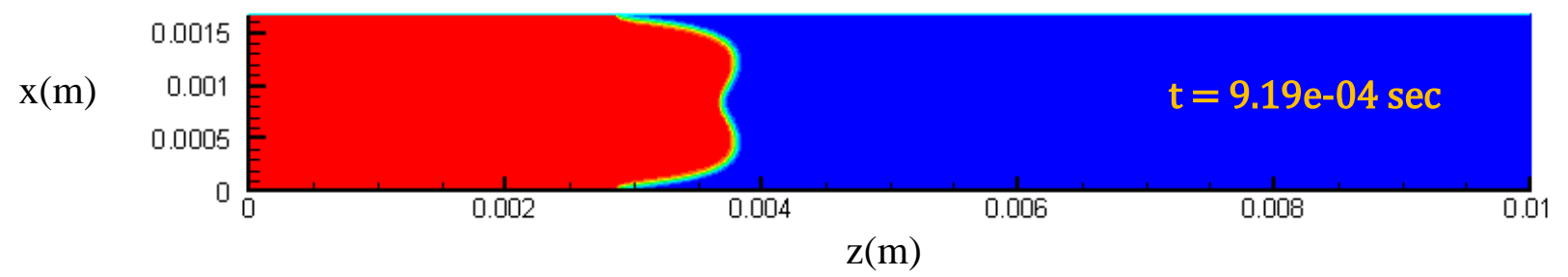

Figure 5.15: A temperature snapshot for a flame with $\mathrm{Le}=2.0$ and $\mathrm{Re}=20$.

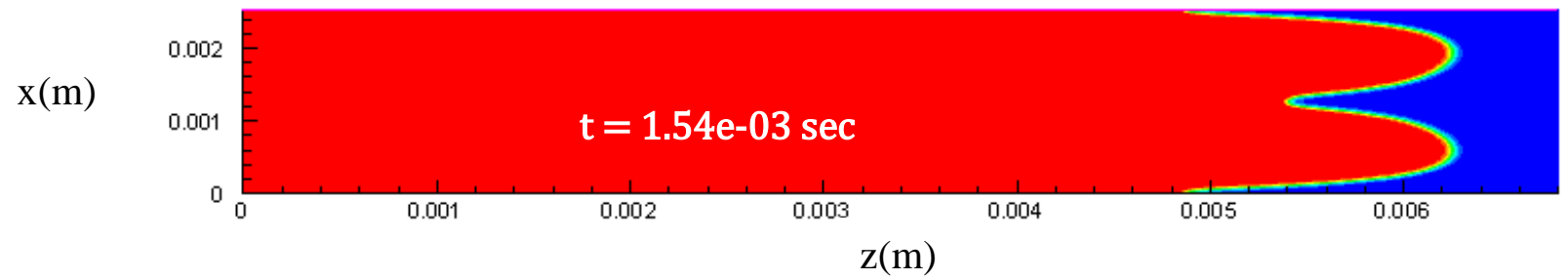

Figure 5.16: A temperature snapshot for a flame with $\mathrm{Le}=1.4$ and $\mathrm{Re}=30$. 


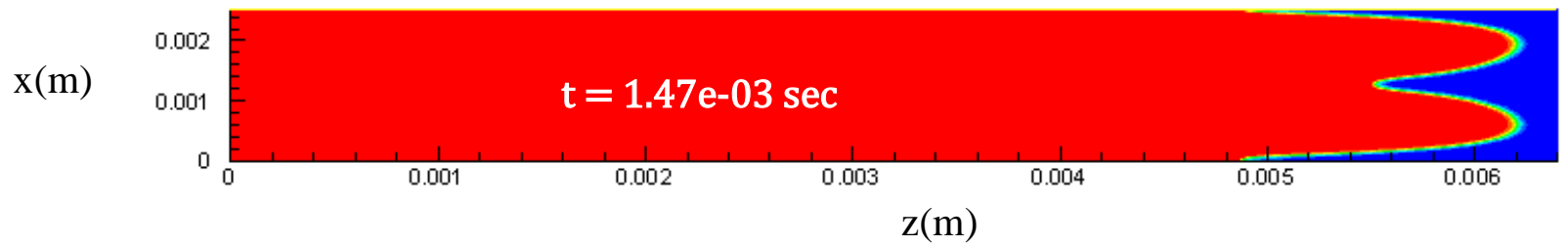

Figure 5.17: A temperature snapshot for a flame with $\mathrm{Le}=2.0$ and $\mathrm{Re}=30$.

After quantifying and analyzing the flame dynamics, we see that there is a crest formation but not to an extent that the flame splits. Additionally, the trough is smooth and more prominent as the flame Reynolds number increases, but there is no major abrupt change in the flame properties. Indeed, all the properties increase smoothly and thus the plots obtained are also very smooth, without any major bumps and disturbances, unlike the Le $<1$ flames. The main reason can be devoted to the non-equidiffussive effects which are promoted at lower Le, Le $<1$.

In Fig. 5.18, the scaled burning rates, plotted versus the scaled time, are compared for the various Lewis and Reynolds numbers. A linear trend is observed in all cases. As a result, the changes in the flame patterns are observed to be prominent. This deviation from exponential acceleration could be devoted to the growth of the thermal diffusivity. The further scrutinized analysis also revealed that the acceleration rate is reduced for the lower Reynolds numbers.

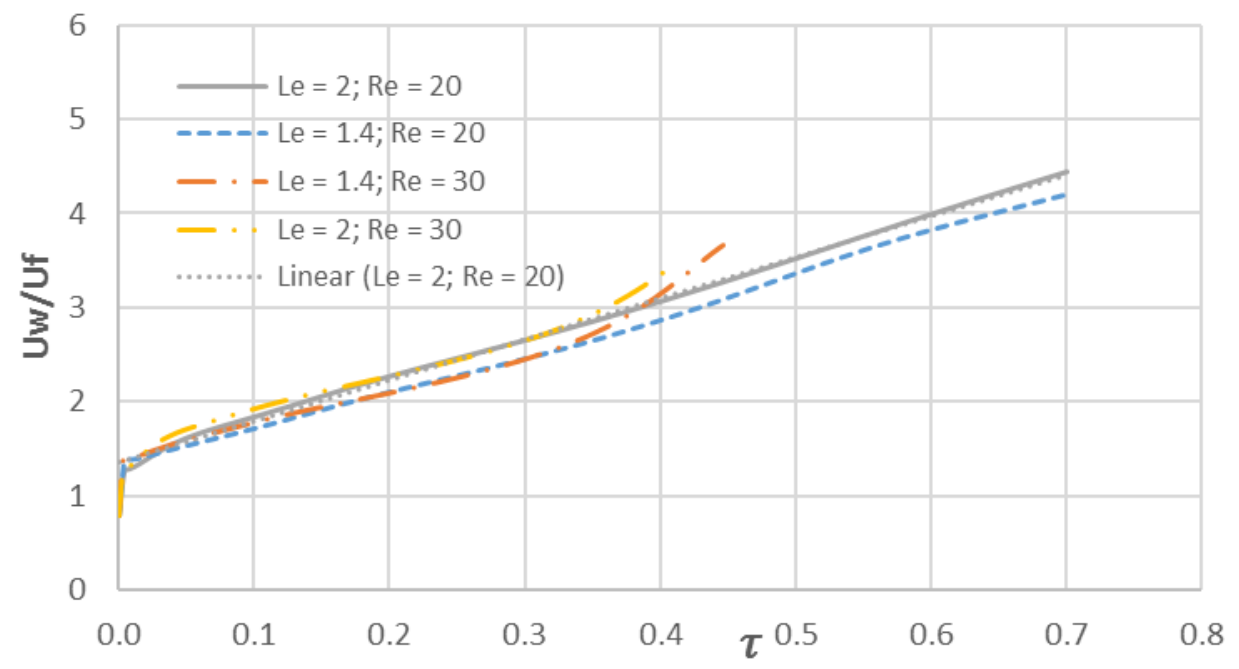

Figure 5.18: The scaled burning rate versus the scaled time for fuel-rich flames. 


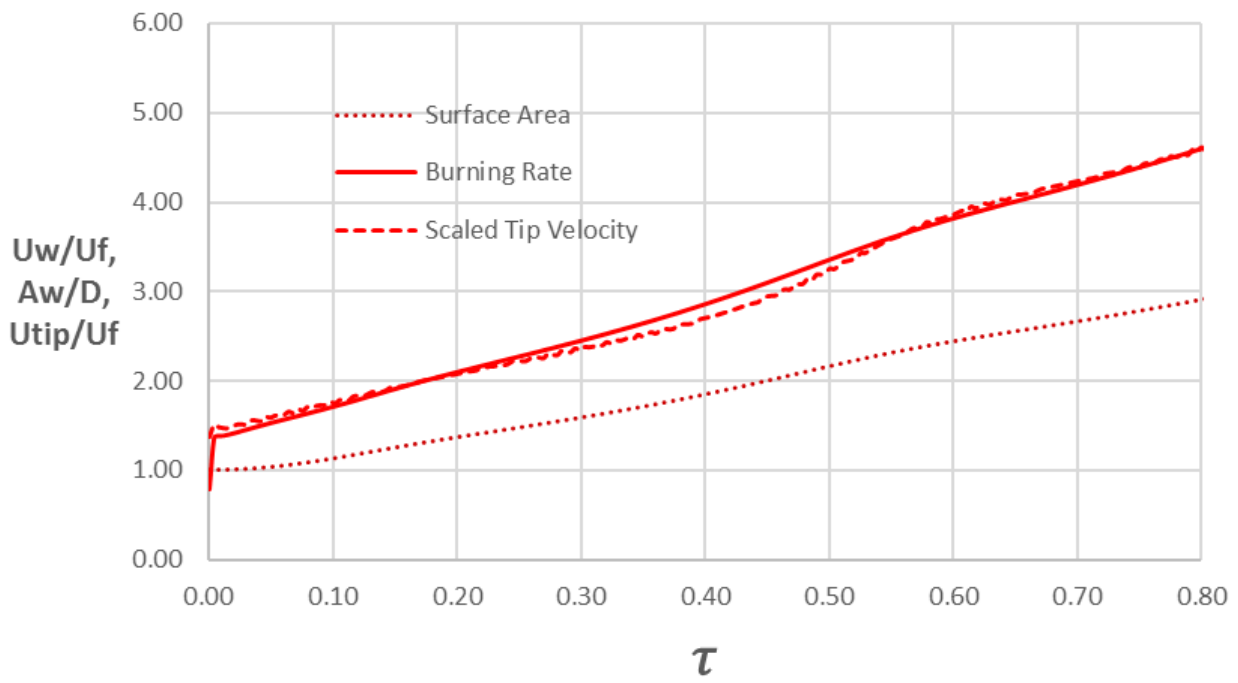

Figure 5.19: The scaled flame properties versus the scaled time for $\mathrm{Le}=1.4$ and $\mathrm{Re}=20$.

In summary, all the flame properties are compared in Fig. 5.19. The scaled flame surface area, the scaled burning rate and the scaled flame tip velocity are interrelated; hence a smooth transition is observed in all of them. In summary, flame acceleration is strengthened with the increase in Re and reduces with Le.

\subsection{Isothermal Walls at Low Temperature}

As the thermal wall conditions have a very remarkable effect on the flame shape and properties, it is important to examine these variables at various conditions. In this subsection, specifically, the isothermal walls with low temperature are investigated. Namely, the wall temperature is kept constant in the simulations, being taken as the room temperature of $300 \mathrm{~K}$. The channels of fixed radius $10 L_{f}$ with various Lewis numbers in the range of $0.2 \leq \mathrm{Le} \leq 2$ are considered. As a result, these set of simulations aim to reveal flame morphology and dynamics in both equidiffusive and non-equidiffusive flames in the case of imposing a cold wall thermal boundary condition.

When the temperature of the channel walls is maintained at $300 \mathrm{~K}$, the heat exchange occurs in two directions: one towards the walls and the other away from the walls, see Fig. 4.4 (the heat flows towards the walls from the burnt matter, being generally at a higher temperature than the walls because of the exothermic nature of combustion), and the other one, where the heat flows from the walls to the cold fuel mixture. 
For the lower Lewis numbers, where the mass diffusion is dominant whereas the effect of thermal diffusivity is relatively minor, the burning rate reads higher values. This facilitates flame acceleration. After some time or after reaching a point that the flame gets cooled down because of the low wall temperature, the burning rate and flame acceleration falls. This occurs because the central hot temperature zone of the burnt matter at the axis is high, which enhances the thermal expansion and leads to the flame contraction as it cools down.

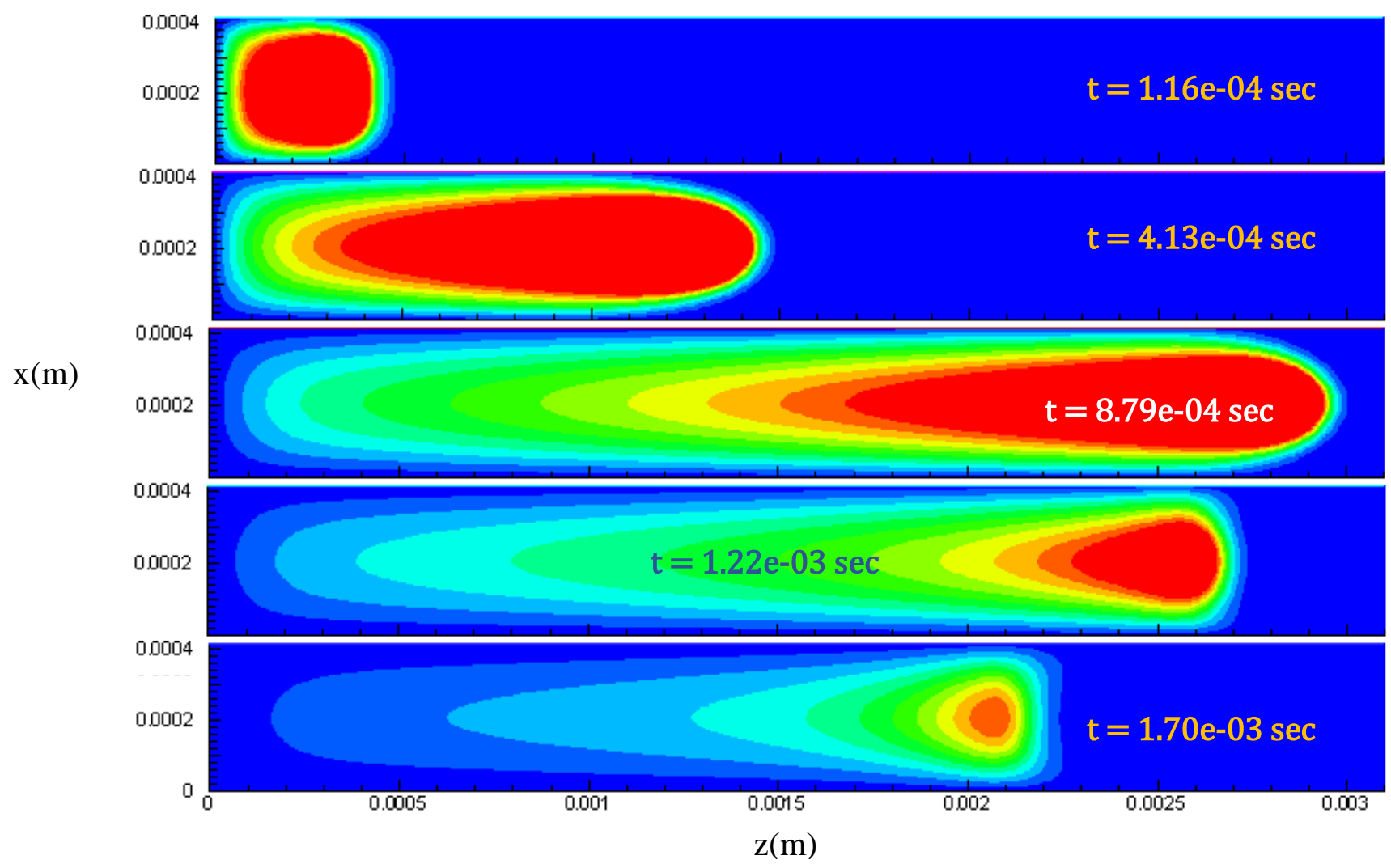

Figure 5.20: The color snapshots of the flame evolution for Le $=0.2$ and $T_{w}=300 \mathrm{~K}$.

The temperature decrease in unburnt matter leads to the decrease in the flame temperature. The burned gas is also cooled to such a stage that the velocity drops drastically, which causes a backward displacement of the flame. This can be seen in the Fig. 5.20 for Le =0.2. As the Le number grows to Le $=1.4$, the similar effects are present but not that clear as we can see in Fig. 5.21. As the reaction further proceeds, the wall effects will become much stronger and the flame elongates leading to sustained acceleration for all Le employed. 


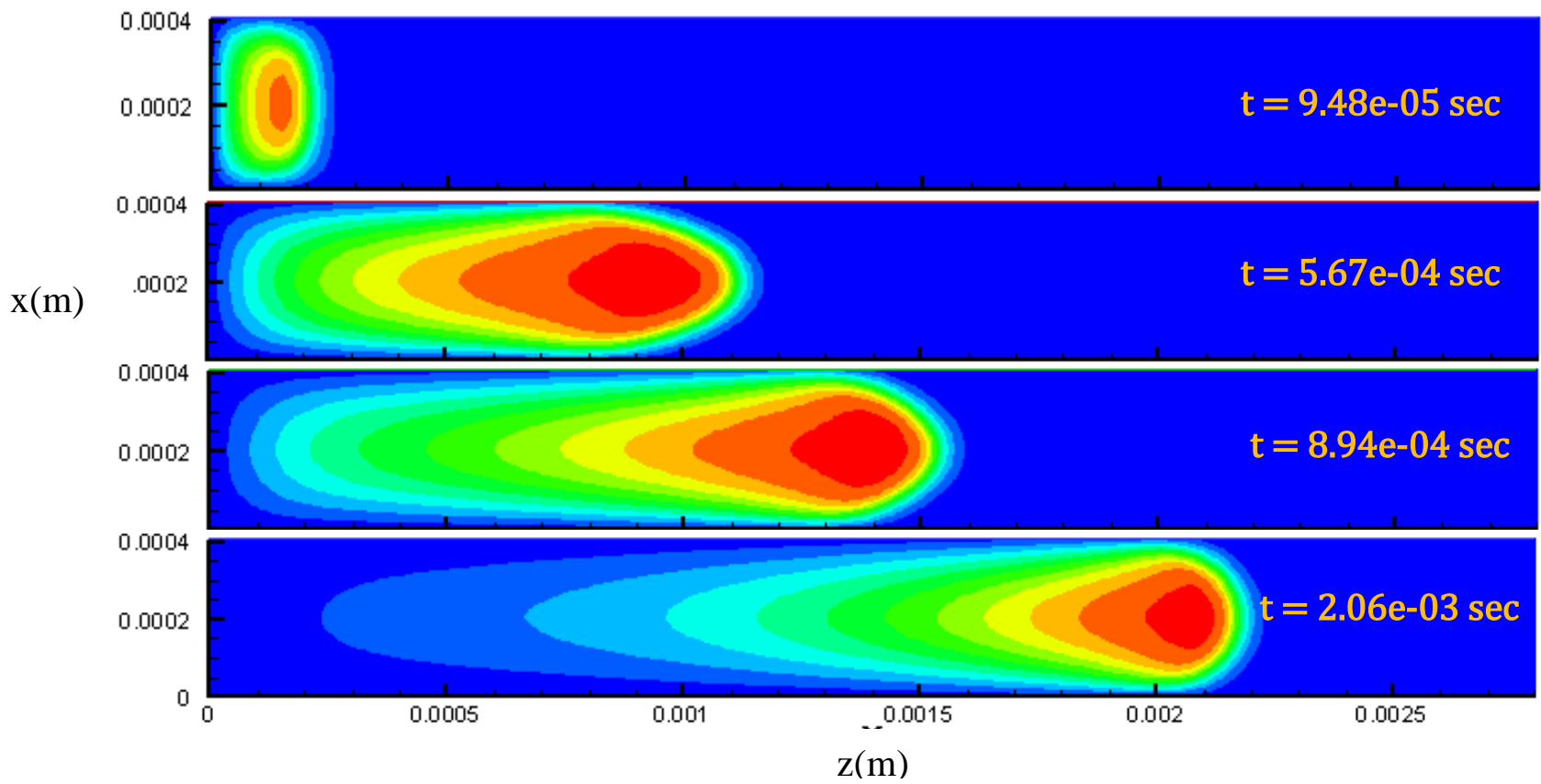

Figure 5.21: The color snapshots of the flame evolution for Le $=1.4$ and $T_{w}=300 \mathrm{~K}$.

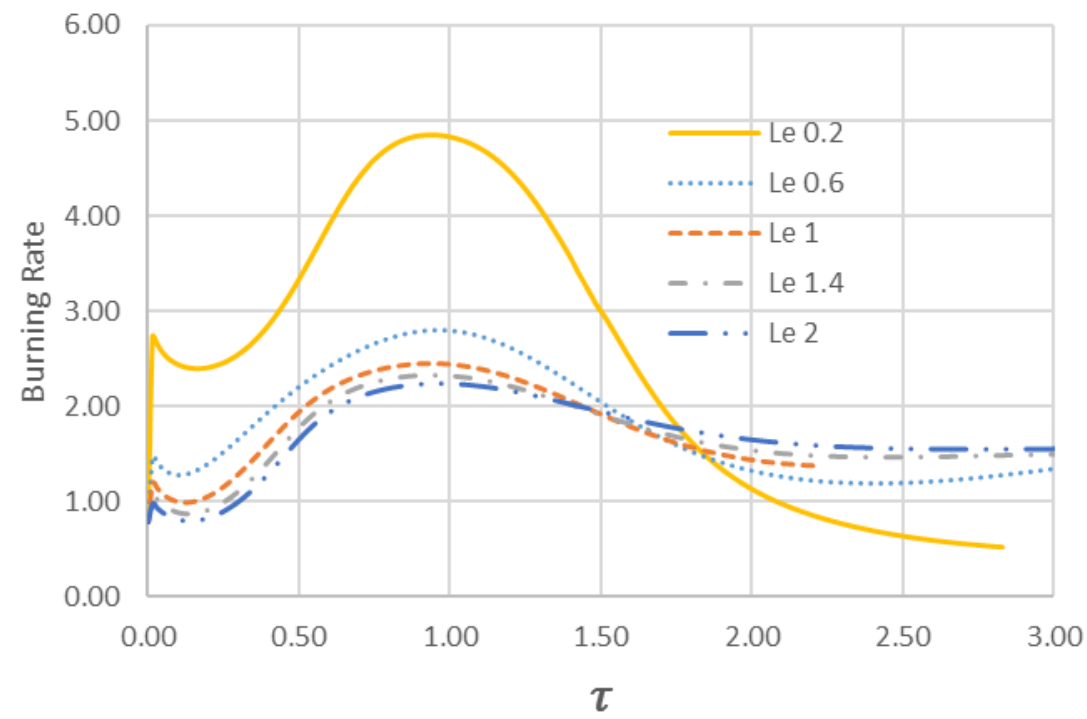

Figure 5.22: Comparison of the burning rate time evolutions for $T_{w}=300 \mathrm{~K}$ and various Le.

A decrease in the burning rate with Le is also observed in Fig. 5.22. But, individually, the burning rate first grows and then decreases as the temperature is reduced. Additionally, as the thermal diffusivity increases, the central core temperature remains intact and the burning rate decreases. But the effect still exists; so the core high temperature region is slightly contracted. Similar qualitative behavior is also observed for the scaled flame tip velocity in Fig. 5.23. 


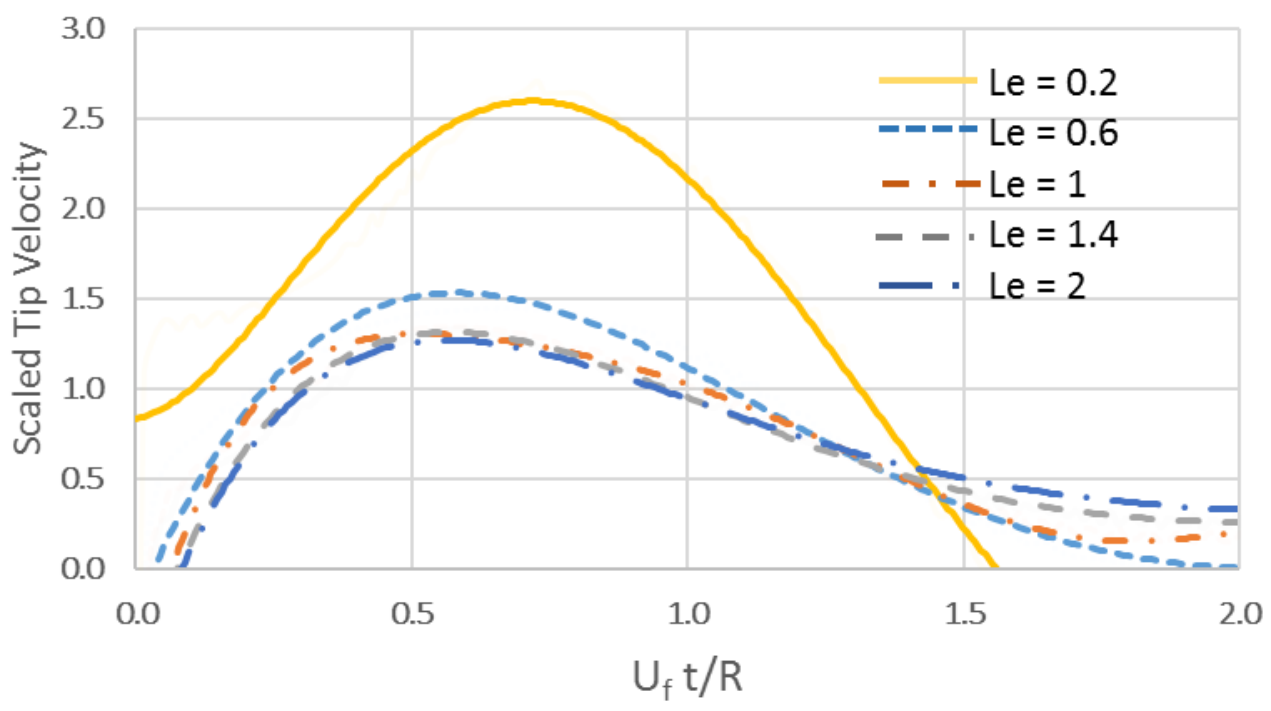

Figure 5.23: The scaled flame tip velocity versus the scaled time for $T_{w}=300 \mathrm{~K}$ and various Le.

\subsection{Isothermal Walls at High Temperature}

In this subsection, we consider isothermal hot walls. Specifically, the wall temperature is $T_{w}=$ $800 \mathrm{~K}$. It is shown that the isothermal hot walls exhibit several interesting features that distinguish the interpretation of the flame acceleration mechanism. Hence, referring to Fig. 5.24 for the flame evolution, some very interesting results are observed related to a flame front position. Specifically, the flame is formed at the walls instead of the centerline as that occurred in the case of cold walls. This makes the flame at center more planar.

The underlying reason for this trend is the hot regions near the walls which facilitate the combustion process; therefore, flame propagation occurs at the walls. It is observed that flame initially starts out as a planar one, but as the walls are at higher temperature the regions near the walls get heated and lead to the pushing of the flame towards the wall. Then the flame at the walls becomes elongated and propagates faster, leaving behind the planar central region. As the propagation continues the central region gets heated up because of the presence of the burnt matter. Due to the thermal expansion, the flame parts merge and become a one part. But the wall effects are still intact and strong enough to elongate the flame at the walls again. This whole flame propagation scenario resembles the closing of a zipper. 


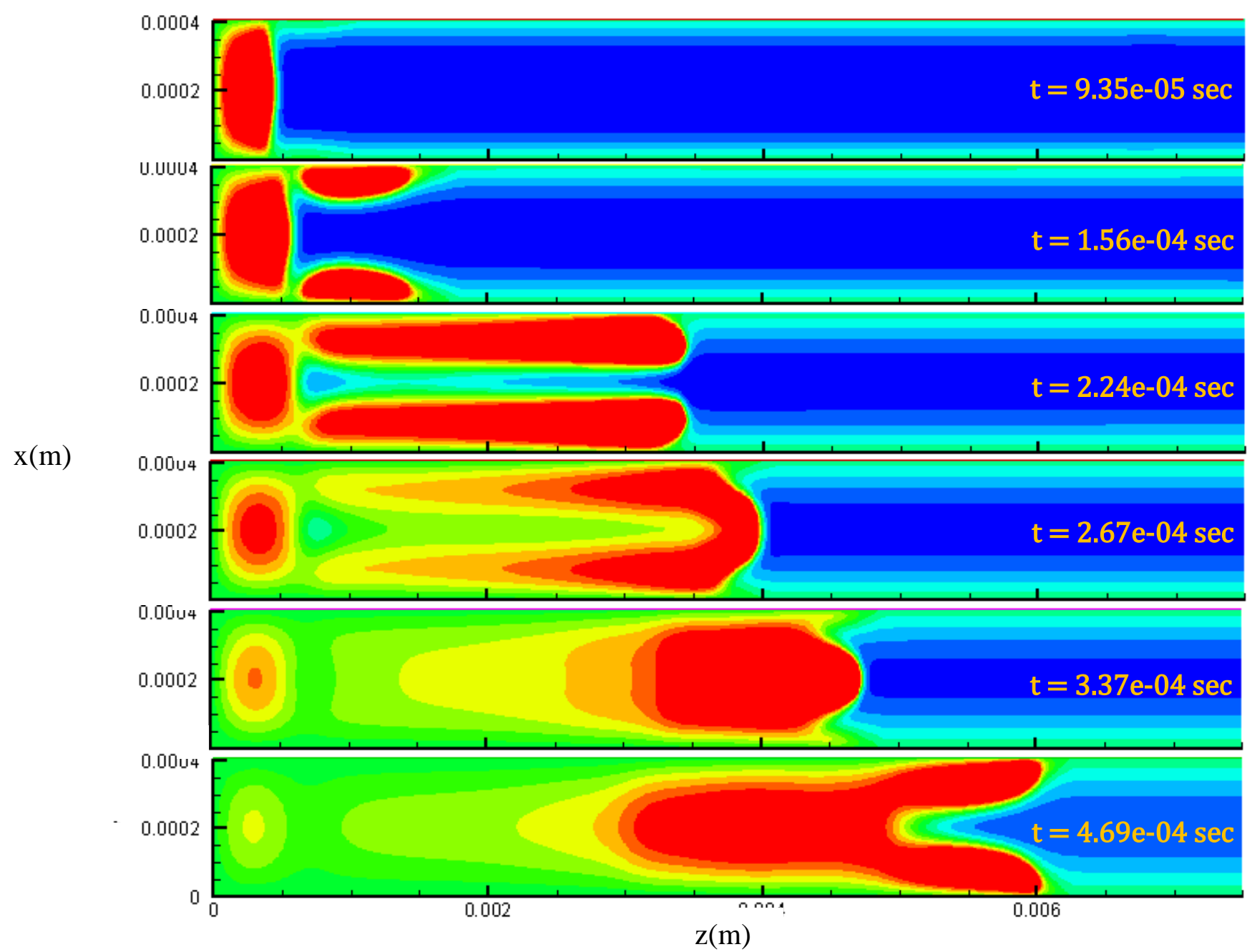

Figure 5.24: The color snapshots of the flame evolution for Le $=0.2$ at $T_{w}=800 \mathrm{~K}$.

In Fig. 5.25, the burning rate, the flame surface area and the scaled tip velocity are compared at the various Lewis numbers in the range $0.2 \leq \mathrm{Le} \leq 2.0$, a constant $\mathrm{Re}=5$, and with isothermal walls of temperature $T_{w}=800 \mathrm{~K}$. As a result, it is observed that scaled burning rate, surface area and flame tip velocity are reduced by the increase in the Lewis number. The effect of thermal diffusivity can be shown as being responsible for such a behavior. It is also observed that the effect of thermal expansion is less apparent for the isothermal wall conditions. As there is heat exchange, the heat loss provides support for combustion extinction. 
(a)

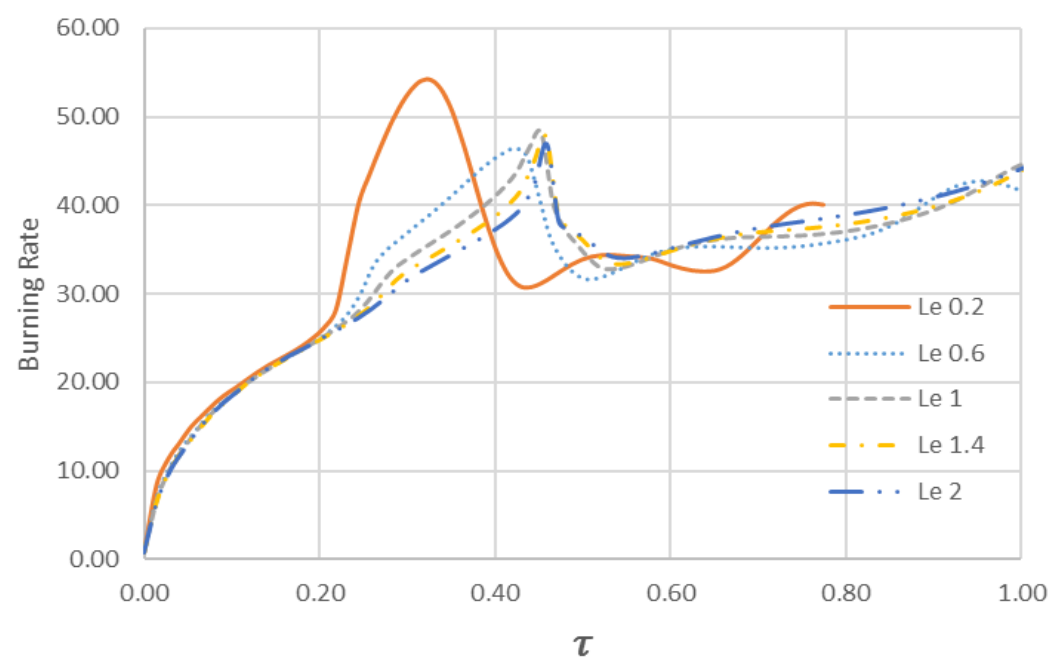

(b)
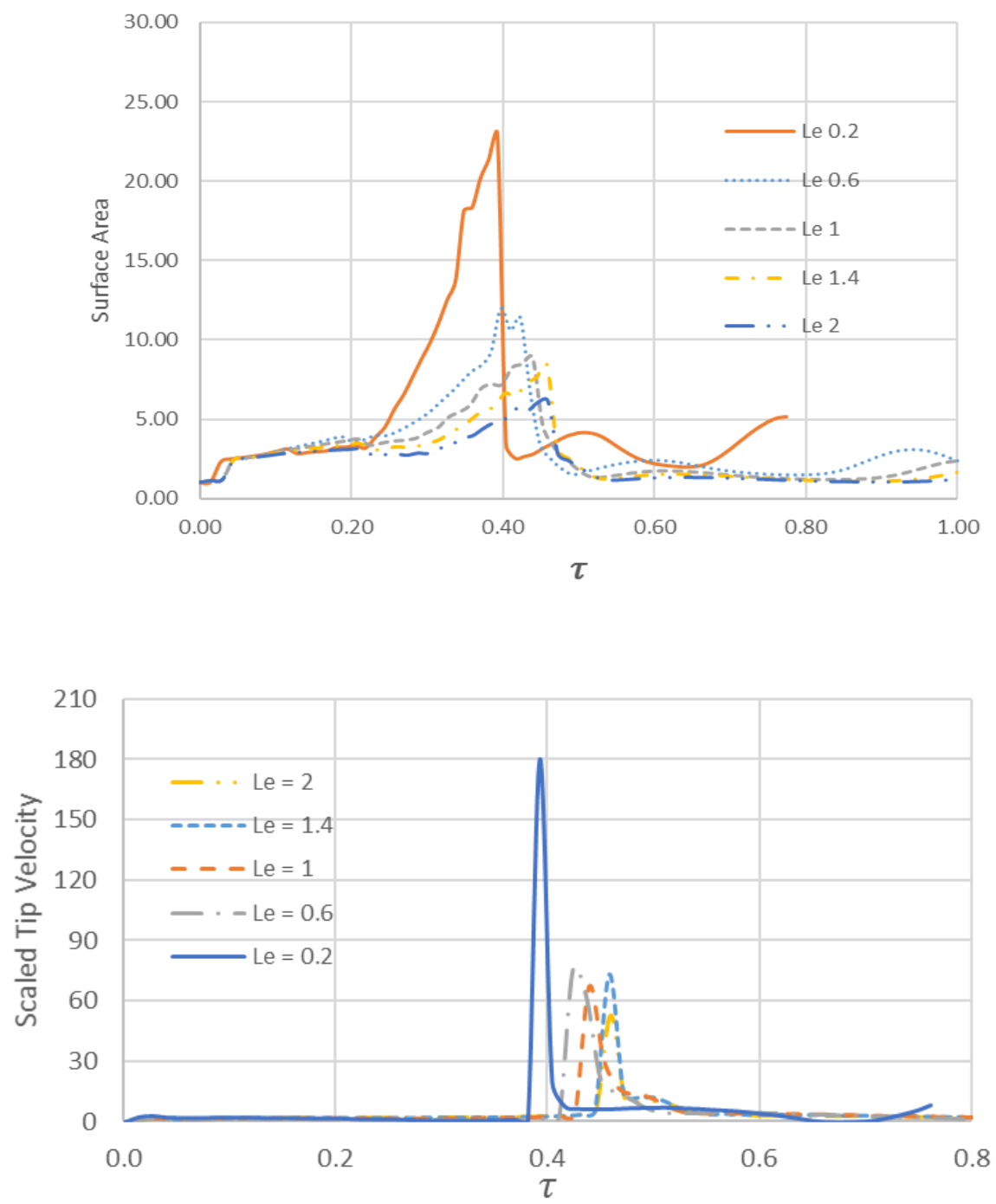

Figure 5.25: The scaled burning rate $U_{w} / U_{f}(\mathrm{a})$, the scaled flame surface area $A_{w} / D(\mathrm{~b})$ and the scaled tip velocity $U_{\text {tip }} / U_{f}$ versus the scaled time $\tau=U_{f} t / R$ for various Le $=0.2 \sim 2.0$. 
In summary, preheated isothermal walls facilitate the flame elongation through the walls that increases the surface area slightly. Along with the presence of very high temperatures in and around the channel, this influences the burning rate to become tremendously high. Once the flame becomes whole again, the flame front is no more extended towards the walls, the surface area drops suddenly and remains almost constant. This happens as Le increases, whereas the burning rate keeps increasing with a slight slope or increment.

In conclusion, flame propagation in isothermal channels can also be described in four stages. The first stage corresponds to no propagation or extinction of the flame, followed by linear acceleration, which is shadowed by the sudden increase in acceleration and finally comes the oscillatory stage, where the velocity keeps oscillating as shown in Fig 5.26.

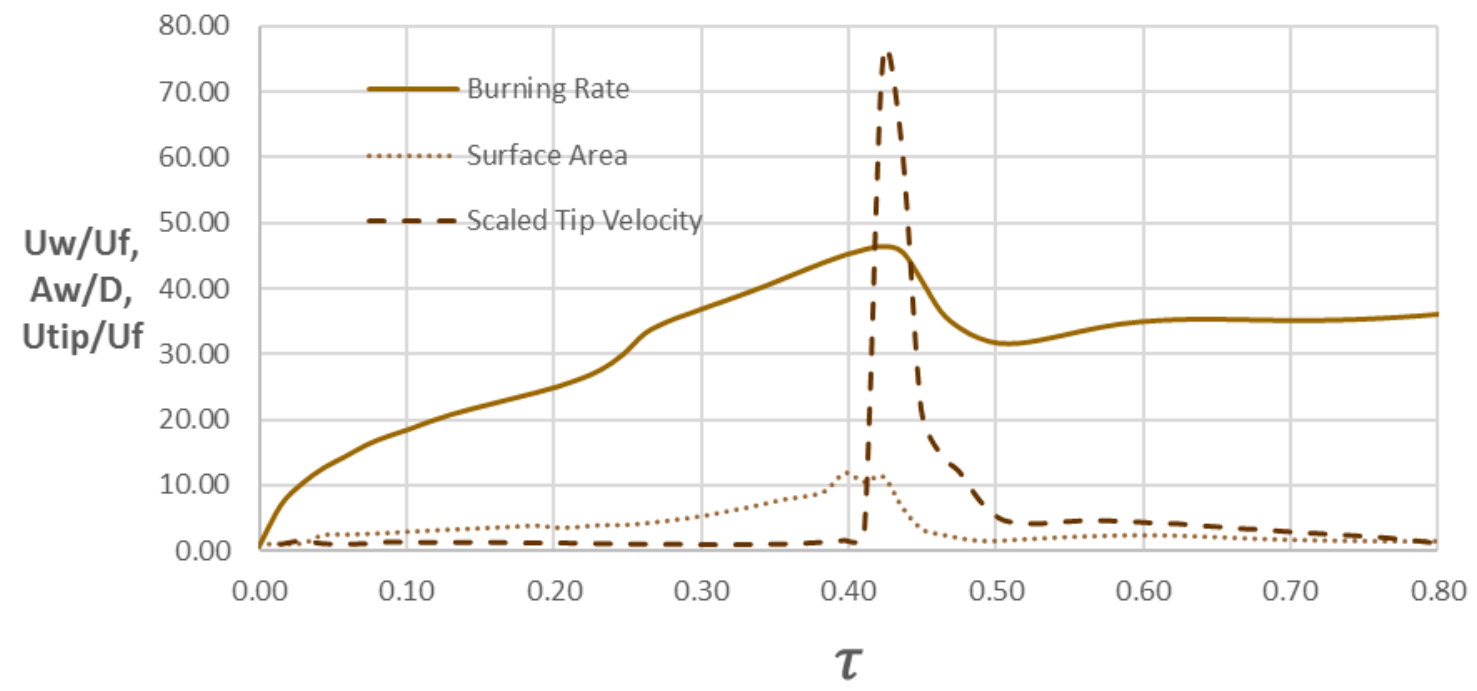

Figure 5.26: Time evolution of the flame properties for Le $=0.6$ with $T_{w}=800 \mathrm{~K}$. 


\section{Chapter 6: Conclusion}

The role of thermal boundary conditions in the flame dynamics in pipes have been investigated by means of computational simulations. The variables explored in this parametric study include the thermal wall conditions with interplay between the thermal and the mass diffusivities in channels of various widths. The numerical simulations are performed for the complete set of combustion equations including thermal conduction, viscosity, diffusion and one-step Arrhenius chemical kinetics.

First, the channels with adiabatic walls were employed and the effect of the Lewis numbers (in the range of $0.2 \leq \mathrm{Le} \leq 2.0$ ) and of the Reynolds numbers (in the range of $5 \leq \operatorname{Re} \leq 30$ ) were investigated. Then the wall conditions were changed to isothermal, with both cold ( $\left.T_{w}=300 \mathrm{~K}\right)$ and preheated $\left(T_{w}=800 \mathrm{~K}\right)$ walls being considered. For all the analyses, we used the thermal expansion factor $\Theta=5$. The outcomes of the simulations include the record of the various flame characteristics such as the flame tip velocity, the burning rate and flame front surface area. Their time evolutions have been identified in all the cases.

When adiabatic channel walls are imposed, the flame exhibits some trough formations as the $\mathrm{Re}$ increases for equidiffusive $(\mathrm{Le}=1)$ flames. However, in case of non-equidiffusive $(\mathrm{Le} \neq 1)$ burning, as the Lewis number decreases, we observe a third trough formation on the flame front, whose intensity increases with Re. The burning rate is also correlated with the third crest and increases accordingly, thereby influencing the rest of the parameters.

However, it is noticed that the isothermal conditions can produce a corrugation of the flame front independent of the non-slip wall conditions. The latter is the result of forcing the walls to maintain certain temperature. This facilitates the burning rate initially, and then terminates as the flame propagates much further.

At low-temperature isothermal walls, the initial flame elongation is similar to the adiabatic conditions but they stop stretching with time and remain steady after that. At high-temperature isothermal walls, the flame corrugation is reduced such that the flame stretches along the walls, and later it expands vertically filling the channel completely. The flame velocities obtained in the isothermal pipes were observed to be much slower than that were obtained in adiabatic pipes. 
Specifically, while adiabatic walls provide the conditions for an exponential increase in the flame velocity with time, the isothermal walls allow at most linear acceleration.

The study of the flame morphology at different conditions helps us to better understand the flame physics. By understanding this, we can use it to our advantage. Namely, we can select the specified conditions to alter the acceleration trends of the flame to match a particular application. For example, if exponential acceleration is desired, one can adopt the adiabatic walls with the lower Lewis numbers. The flame speed in the combustors can be manipulated to our advantage by knowing the effect of each parameter.

The variation of the channel geometry also influences the flame dynamics, which can be applied in nanotubes to control the speed of the thermal waves. At the same time, changing the equivalence ratio of the fuel-air mixture can modify the laminar flame speed $U_{f}$, which can be constructively used in micro-combustors. Flame acceleration can also be controlled by varying the wall temperature. Namely, the higher the temperature, the higher acceleration is. One can simultaneously heat/cool the walls to manipulate acceleration and suit a particular application.

This study can potentially be extended to numerous directions. In particular, the walls can be incorporated with a variable heat transfer flux or there may be obstacles added into the path of the flame inside the channel. This study can also be extended to different geometries other than channels. Apart from the simulations, an analytical model for linear flame acceleration is desired. 


\section{References}

[1] "- Flame, Steam, Fuel, and Engines," Combustion - Applications, [Online]. Available: "http://science.jrank.org/pages/1631/Combustion-Applications.html">Combustion Applications $</ \mathrm{a}\rangle$. [Accessed 28 October 2016].

[2] O. Elaine, V. Gamezo, "Origins of the deflagration-to-detonation transition in gas-phase combustion," Combustion and Flame, vol. 148, p. 4, 2007.

[3] Y. Zeldovich, D. Frank-Kamenetski, "A Theory of Thermal Propagation of Flame," ACTA Physico-Chimica URSS, vol. 9(2), p. 341, 1938.

[4] C.K. Law, Combustion Physics, New York: Cambridge University Press, 2006.

[5] W. Choi, S. Hong, J. Abrahamson, J.-H. Han, C. Song, N. Nair, S. Baik and M. Strano, "Chemically driven carbon-nanotube-guided thermopower waves," Nature Materials, vol. 9, p. 423, 2010.

[6] Y. Ju, K. Maruta, "Microscale combustion: Technology development and fundamental research," Progress in Energy and Combustion Science, vol. 37, p. 669, 2011.

[7] S. Rockwell, A. Rangwala, "Influence of coal dust on premixed turbulent methane-air flames," Combustion and Flame, vol. 160, p. 635, 2013.

[8] Y. Suzuki, Y. Okada, J. Ogawa, S. Sugiyama, T. Toriyama, "Experimental study on mechanical power generation from MEMS internal combustion engine," Sensors and Actuators A: Physical, vol. 141, no. 2, p. 654, 2008.

[9] L. Kagan, G. Sivashinsky, "The transition from deflagration to detonation in thin channels," Combustion and Flame, vol. 134, p. 389, 2003.

[10] V. Akkerman, C.K. Law, V. Bychkov, L.-E. Eriksson, "Analysis of flame acceleration induced by wall friction in open tubes," Physics of Fluids, vol. 22, no. 5, p. 053606, 2010.

[11] L. Landau, E. Lifshitz, Fluid Mechanics, Oxford: Pergamon Press, 1987.

[12] Y. Zeldovich, G. Barenblatt, V. Librovich, G. Makhviladze, The Mathematical Theory of Combustion and Explosions, New York: Consultants Bureau, 1985.

[13] V. Bychkov, A. Petchenko, V. Akkerman, L.-E. Eriksson, "Theory and modeling of accelerating flames in tubes," Physical Review E, vol. 72, p. 046307, 2005.

[14] C. Clanet, G. Searby, "On the "Tulip Flame" Phenomenon," Combustion and Flame, vol. 105, p. 225, 1996.

[15] V. Bychkov, V. Akkerman, G. Fru, A. Petchenko, L.-E. Eriksson, "Flame Acceleration at the Early Stages of Burning in Tubes," Combustion and Flame, vol. 150, p. 263, 2007.

[16] K. Shelkin, "Influence of the Wall Roughness on Initiation and Propagation of Detonation in Gases," Zh. Eksp. Teor. Fiz., vol. 10, p. 823, 1940.

[17] V. Akkerman, V. Bychkov, A. Petchenko, L.-E. Eriksson, "Flame oscillations in tubes with nonslip at the walls," Combustion and Flame, vol. 145, p. 675, 2006.

[18] D. Valiev, V. Bychkov, V. Akkerman, L.-E. Eriksson, M. Marklund, "Heating of the fuel mixture due to viscous stress ahead of accelerating flames in deflagration-to-detonation transition," Physics Letters A, vol. 372, no. 27-28, p. 4850, 2008.

[19] D. Valiev, V. Bychkov, V. Akkerman, L.-E. Eriksson, "Different stages of flame acceleration from slow burning to Chapman-Jouguet deflagration," Physical Review E, vol. 80, p. 036317, 2009. 
[20] V. Bychkov, V. Akkerman, D. Valiev, C.K. Law, "Role of compressibility in moderating flame acceleration in tubes," Physical Review E, vol. 81, p. 026309, 2010.

[21] S. Bilgili, "Acceleration of non-equidiffusive flames in vhannels: computational simulations and analytical studies," MS Theis, West Virginia University, Morgantown, 2015.

[22] M. Liberman, V. Bychkov, "Dynamics and stability of premixed flames," Physics Reports, vol. 325, no. 4-5, p. 115, 2000.

[23] G. Barenblatt, Y. Zeldovich, Prikl. Mat. Mekh., vol. 21, no. 6, p. 856, 1959.

[24] J. Rosen, "Theory of laminar flame stability. I. Analytic stability condition," The Journal of Chemical Physics, vol. 22, no. 4, p. 733, 1954.

[25] B. Lewis, G. von Elbe, Combustion, Flames, and Explosion of Gases, New York, 1938.

[26] K. Sabdenov, "Diffusion-thermal instability of a laminar flame," Journal of Engineering Physics and Thermophysics, vol. 75, no. 4, p. 859, 2002.

[27] G. Darrieus, "Propagation d'un front de flamme," La Technique Moderne, 1938.

[28] L. Landau, "On the theory of slow combustion," Acta Physicochimica URSS, vol. 19, p. 77, 1944.

[29] P. Pelce, P. Clavin, "Influence of hydrodynamics and diffusion upon the stability limits of laminar premixed flames," Journal of Fluid Mechanics, vol. 124, p. 219, 1982.

[30] M. Matalon, B. Matkowsky, "Flames as gasdynamic discontinuities," Journal of Fluid Mechanics, vol. 124, p. 239, 1982.

[31] V. Akkerman, V. Bychkov, "Velocity of weakly turbulent flames of finite thickness," Combustion Theory and Modelling, vol. 9, no. 2, p. 323, 2005.

[32] G. Searby, J. Quinard, "Direct and indirect measurements of Markstein numbers of premixed flames," Combustion and Flame, vol. 82, no. 3-4, p. 298, 1990.

[33] S. Davis, J. Quinard, G. Searby, "Markstein numbers in counterflow, methane- and propaneair flames: a computational study," Combustion and Flame, vol. 130, no. 1-2, p. 123, 2002.

[34] R. Pitz, S. Hub, P. Wang, "Tubular premixed and diffusion flames: effect of stretch and curvature," Progress in Energy and Combustion Science, vol. 42, p. 1, 2014.

[35] C. Hackert, J. Ellzey, O. Ezekoye, "Effects of thermal boundary conditions on flame shape and quenching in ducts," Combustion and Flame, vol. 112, p. 73, 1998.

[36] J. Daou, M. Matalon, "Influence of conductive heat losses on the propagation of premixed flames in channels," Combustion and Flame, vol. 128, p. 321, 2002.

[37] D. Norton, D. Vlachos, "Combustion characteristics and flame stability at the microscale: a CFD study of premixed methane/air mixtures," Chemical Engineering Science, vol. 58, p. 4870, 2003.

[38] L. Kagan, D. Valiev, M. Liberman, V. Gamezo, E. Oran, G. Sivashinsky, "Effect of hydraulic resistance and heat losses on the deflagration-to-detonation transition.," in Pulse Detonation Engine, Moscow, Torus Press Ltd, p. 51, 2006.

[39] O. Ugarte, "Effect of boundary conditions on propagation and morphology of premixed flames in narrow conduits," PhD Disseration, West Virginia University, Morgantown, 2015.

[40] H. Xiao, Q. Wang, X. He, J. Sun, X. Shen, "Experimental study on the behaviors and shape changes of premixed hydrogen-air flames propagating in horizontal duct," International Journal of Hydrogen Energy, vol. 36, no. 10, p. 6325, 2011. 
[41] V. Bychkov, S. Golberg, M. Liberman, L.-E. Eriksson, "Propagation of curved stationary flames in tubes," Physical Review E, vol. 54, p. 3713, 1996.

[42] O. Travnikov, V. Bychkov, M. Liberman, "Numerical studies of flames in wide tubes: Stability limits of curved stationary flames," Physical Review E, vol. 61, p. 468, 2000.

[43] V. Akkerman, B. Demirgok, V. Bychkov, D. Valiev, C.K. Law, M-H. Wu, "Analysis of ethylene-oxygen combustion in micro-pipes," in 9th U.S. National Combustion Meeting, Cincinnati, OH, May 17-20, 2015.

[44] G. Roy, S. Frolov, A. Borisov, D. Netzer, "Pulse detonation propulsion: challenges, current status, and future perspective," Progress in Energy and Combustion Science, vol. 30, p. 545, 2004.

[45] B. Demirgok, O. Ugarte, D. Valiev, V. Akkerman, "Effect of thermal expansion on flame propagation in channels with non-slip walls," Proceedings of the Combustion Institute, vol. 35, p. 929, 2015.

[46] D. Valiev, V. Akkerman, M. Kuznetsov, L. Eriksson, C.K. Law, "Influence of gas compression on flame acceleration in the early stage of burning in tubes," Combustion and Flame, vol. 160, no. 1, p. 97, 2013.

[47] G. Barenblatt, Y. Zeldovich, A. Istratov, "Thermodiffusional stability of a laminar flame," Prikl. Mekh. Tekh. Fiz., vol. 4, p. 21, 1962.

[48] Y. Kanel, "Certain Problems relative to some equations in theory of burning," Dokl. Akad. Nauk SSSR, vol. 2, p. 277, 1961. 